\title{
INVASIVE ALIEN SPECIES OF UNION CONCERN (REGULATION 1143/2014) IN CROATIA
}

\section{Igor Boršić*, Ana Ješovnik, Tanja Mihinjač, Petra Kutleša, Sandra Slivar, Martina Cigrovski Mustafić \& Sonja Desnica}

\author{
Croatian Agency for Environment and Nature, Radnička cesta 80/7, HR-10000 Zagreb, Croatia
}

Boršić, I., Ješovnik, A., Mihinjač, T., Kutleša, P., Slivar, S., Cigrovski Mustafić, M. \& Desnica, S.: Invasive Alien Species of Union Concern (Regulation 1143/2014) in Croatia. Nat. Croat., Vol. 27, No. 2, 357-398, Zagreb, 2018.

Regulation 1143/2014 on the prevention and management of the introduction and spread of invasive alien species creates the framework for the prevention, minimization, and mitigation of the harmful effects of invasive alien species on biodiversity in the European Union. Commission Implementing Regulations 2016/1141 and 2017/1263 delivered the list of invasive alien species of Union concern ("the Union list"). Out of 49 species on the Union list, 17 species are present in Croatia. They include five plants (Plantae), three crustaceans (Crustacea), two freshwater fish (Actinopterygii), one reptile (Reptilia), one bird (Aves), and five mammals (Mammalia). Their systematics and biology, native and introduced range, history of introduction, pathways of introduction to Croatia, impact in the introduced range, including any known impacts in Croatia, are presented. Furthermore, their distribution in Croatia, based on the available data, is shown on the distribution maps.

Key words: non-native species, Croatia, distribution, fauna, flora

Boršić, I., Ješovnik, A., Mihinjač, T., Kutleša, P., Slivar, S., Cigrovski Mustafić, M. \& Desnica, S.: Invazivne strane vrste koje izazivaju zabrinutost u Uniji (Uredba 1143/2014) prisutne u Hrvatskoj. Vol. 27, No. 2, 357-398, Zagreb, 2018.

Uredba 1143/2014 o sprječavanju i upravljanju unošenja i širenja invazivnih stranih vrsta određuje okvir za sprječavanje, svođenje na najmanju moguću mjeru i ublažavanje štetnih učinaka invazivnih stranih vrsta na bioraznolikost u Europskoj uniji. Provedbenim uredbama Komisije 2016/1141 i 2017/1263 donesen je popis invazivnih stranih vrsta koje izazivaju zabrinutost u Uniji ("Unijin popis"). Od ukupno 49 vrsta na Unijinom popisu, 17 vrsta je prisutno u Hrvatskoj. One uključuju pet biljaka (Plantae), tri raka (Crustacea), dvije slatkovodne ribe (Actinopterygii), jednog gmaza (Reptilia), jednu pticu (Aves) i pet sisavaca (Mammalia). Ovdje predstavljamo njihovu sistematiku i biologiju, prirodno područje rasprostranjenosti i područje unosa, povijest unosa, putove unosa u Hrvatsku, utjecaj u području unosa, uključujući i poznate utjecaje u Hrvatskoj. Također, njihova rasprostranjenost u Hrvatskoj, temeljem dostupnih podataka, prikazana je kartama rasprostranjenosti.

Ključne riječi: fauna, flora, Hrvatska, rasprostranjenost, strana vrsta 


\section{INTRODUCTION}

Regulation 1143/2014 on the prevention and management of the introduction and spread of invasive alien species, which entered into force on 1 January 2015, systematically deals with the problem of invasive alien species (IAS) at the level of the European Union (EU, 2014). This Regulation is binding in its entirety and directly applies to all Member States, including Croatia. It sets out rules to prevent, minimize, and mitigate the harmful effects of invasive alien species on biodiversity in the European Union. The framework for its implementation in Croatian legislation is set by the new Act on the Prevention and Management of the Introduction and Spread of Alien and Invasive Alien Species (ANONYMOUs, 2018) and its subordinate legislation.

According to the Regulation, the Commission delivers a list of invasive alien species of Union concern ("the Union list"). To be considered for the list each species must be proposed by the European Commission or one of the Member States, which then conducts a comprehensive risk assessment. The risk assessment is a document that contains description of the species' taxonomy, distribution and ecology, the possible pathways of its introduction and spread, known and potential adverse impacts on biodiversity, ecosystem services, human health, safety and economy, including the assessment of possible benefits. The assessed alien species can be added to the list if it meets a predefined set of criteria, such as: it must be able to establish a viable population and spread, it needs to be likely that it would have a significant adverse impact on biodiversity, related ecosystem services, human health or the economy, and it has to be likely that listing the species on the Union list will effectively prevent, reduce or mitigate these adverse effects. Furthermore, it has to be approved by the IAS Committee. After the listing of a species on the Union list, its import, trade, breeding, growing, and keeping are restricted (EU, 2014).

The Regulation also sets measures to combat the negative impact of invasive alien species through prevention, early detection, and rapid eradication. This approach, which focuses on prevention and eradication measures in early stages of invasion, is based on numerous studies that show that eradication or control of IAS after the population has established and reached a certain size is either exceedingly hard or impossible, and often accompanied with large economic losses (Veitch \& Clout, 2002; ANDERSON, 2005).

The Union list must be comprehensively reviewed at least every six years, and in the meantime new species can be added or removed according to the procedures prescribed in the Regulation (EU, 2014). The first list of invasive alien species of Union concern, adopted by Commission Implementing Regulation (EU) 2016/1141 of 13 July 2016 (EC, 2016), included a total of 37 species. On 12 July 2017, 12 more species were added to the Union list by Commission Implementing Regulation (EU) 2017/1263 (EC, 2017), bringing the current number of species on the Union list to 49 (Tab. 1).

In this paper we provide baseline data about the invasive alien species from the Union list currently known in Croatia, including their distribution, history of introduction, biology, and impact on biodiversity. Although there are other invasive alien species in Croatia, they are not included on the Union list and therefore not in the scope of this paper. 


\section{MATERIALS AND METHODS}

\section{Study area}

Croatia is a south-eastern European country situated on the eastern coast of the Adriatic Sea, with a land area of $56,594 \mathrm{~km}^{2}$ and a sea area of $31,067 \mathrm{~km}^{2}$. It spans three biogeographical regions: Mediterranean, Alpine, and Continental (EEA, 2016). The Continental biogeographical region is predominantly flat, with the floodplains of the Sava, Mura, Drava, and Danube rivers. The Alpine biogeographical region contains geomorphologically diverse Dinaric Mountains (highest elevation 1831 meters) dominated by fir and beach forests. The Mediterranean biogeographical region comprises the eastern Adriatic coast and the islands. The mean annual temperature in Croatia ranges from $3^{\circ} \mathrm{C}$ in the mountain areas, to $17^{\circ} \mathrm{C}$ along the coast and on the islands. The mean annual amount of precipitation ranges from $300 \mathrm{~mm}$ on the outer southern Adriatic islands (the island of Palagruža) to over $3500 \mathrm{~mm}$ in the mountain region (Gorski kotar, Velebit Mountain) and the Konavle Plain (Zaninović et al., 2008).

\section{Terminology}

The terminology used throughout the manuscript is adapted from RicHARDson (2000), Copp et al. (2005), Regulation 1143/2014 (EU, 2014), and NOBANIS (2015):

Alien species (syn. non-native, non-indigenous, exotic) - any species found outside its natural range.

Casual species - an alien species that is found only occasionally, and may occasionally reproduce, but is still unable to form self-replacing populations. Its presence in introduced range relies on repeated introductions.

Door knocker species - an alien species that is not yet introduced or established, but is expected to arrive in the near future.

Introduction - the human-mediated movement, intentional or unintentional, of a species outside its natural range.

Naturalized species (syn. established) - an alien species that reproduces independently and that is capable of sustaining a viable population in the introduced range.

Invasive alien species (IAS) - an alien species whose introduction or spread has been found to threaten or adversely impact biodiversity and related ecosystem services, human health, and the economy.

Invasive alien species of Union concern - an invasive alien species whose current or potential adverse effects on biodiversity or the related ecosystem services are such that they require coordinated action at Union level.

\section{Species data outline}

All nomenclature follows the implementing regulations (EC, 2016, 2017). When the regulation's nomenclature is not in agreement with the most recent taxonomic changes, we address this accordingly. For species for which Croatian vernacular names do not exist in literature, we devised them from English names. 
For each species we summarize the following: systematics and biology, native and introduced range, history of introduction, introduction pathway to Croatia, species' impact in its introduced range, including any known impacts in Croatia.

We obtained the distribution data for Europe from European and global databases and projects (e. g. NOBANIS, 2017; CABI, 2018a; DAISIE, 2018; EASIN, 2018; GRIIS, 2018), as well as from national check-lists of alien and invasive alien species (e. g. PYŠEK et al., 2012). For plant species existing data from the Flora Croatica Database (Nikolić, 2018) were used. For animal taxa, in addition to the listed databases, we obtained the data from literature and the Croatian Agency for Environment and Nature database (CAEN, 2018). Distribution maps were prepared using ESRI GIS ArcMap 10.1 software. Although the real distribution of some species (e. g. Pseudorasbora parva) in Croatia is probably wider than shown on the distribution maps, only known distribution data are shown.

\section{RESULTS AND DISCUSSION}

Out of 49 invasive alien plant and animal species of Union concern, there are 17 species present in Croatia, including five plant species (one Liliopsida and four Magnoliopsida), three freshwater crayfish (Malacostraca), two freshwater fish (Actinopterygii), one reptile (Reptilia), one bird (Aves), and five mammals (Mammalia) (Tab. 1, Figs. 1, 2). Two additional animal species from the Union list, Lithobates catesbeianus (Amphibia) and Eriocheir sinensis (Malacostraca), are established in neighbouring countries and recorded near the Croatian border, so their presence in Croatia is possible. Furthermore, three plant species from the Union list, Eichhornia crassipes (Martius) Solms, Ludwigia peploides (Kunth) P.H. Raven, and Pennisetum setaceum (Forssk.) Chiov. are not listed in the check list of Croatian vascular flora (Nikolić, 2018) and, to our present knowledge, are not present in the wild in Croatia. However, it is likely that they are present in Croatia as ornamental species in cultivation. The species Eichhornia crassipes and Ludwigia peploides are sold via the internet for aquaria and fish ponds, while Pennisetum setaceum is also on sale by some plant nurseries, despite Regulation restrictions.

\section{PLANTS}

There are five vascular plant species of Union concern present in Croatia: Asclepias syriaca L., Elodea nuttallii (Planch.) St. John, Heracleum mantegazzianum Sommier \& Levier, Impatiens glandulifera Royle, and Myriophyllum heterophyllum Michaux.

Asclepias syriaca L. - common milkweed (Croatian: pravo cigansko perje, prava svilenica); Figs. 1a, 3

Species Asclepias syriaca is a perennial herb up to $150 \mathrm{~cm}$ tall with branched rhizome. Leaves are opposite, simple, entire, ovate-oblong, acute at apex and rounded at base. They are white-pubescent beneath and dark green, sparsely pubescent above. Flowers are entomophilous, 5-merous, actinomorphic, built in the specific way characteristic of the family Asclepiadaceae, with pink corona composed of hoods and horns. Inflorescences are many-flowered terminal or axillary, subglobose to globose umbels. Fruit consists of a pair of white-pubescent, sulcate and spiny follicles, but usually only one follicle develops. Seeds are numerous, flat, brown and with a tuft of silky white hairs apically. They are spread by wind (anemochory). It flowers from June to August (HEYwood, 1972; Petrova et al., 2013; Nikolić et al., 2014). 


\begin{tabular}{|c|c|c|c|c|c|c|c|c|c|c|c|c|c|c|c|c|c|}
\hline 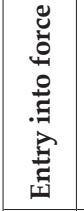 & 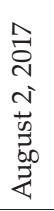 & 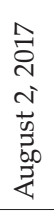 & 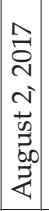 & 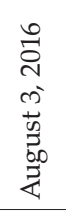 & 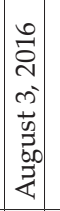 & 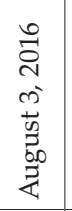 & 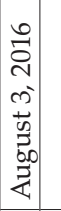 & 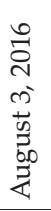 & 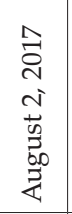 & 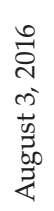 & 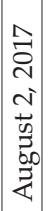 & 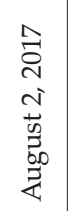 & 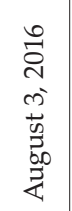 & 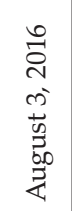 & 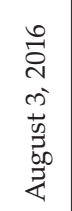 & 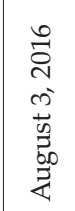 & 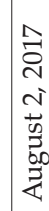 \\
\hline 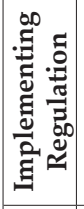 & 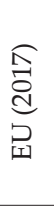 & 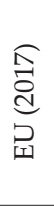 & 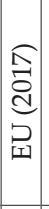 & 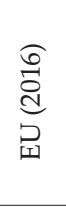 & 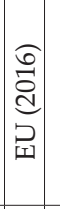 & 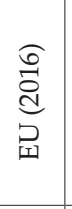 & 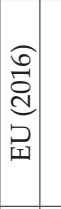 & $\begin{array}{l}0 \\
\stackrel{0}{0}\end{array}$ & స్త్ర & $\begin{array}{l}0 \\
\stackrel{0}{0} \\
\stackrel{d}{d} \\
\stackrel{1}{d}\end{array}$ & $\left|\begin{array}{c}\widehat{\sigma} \\
\vec{D} \\
d \\
己 \\
\end{array}\right|$ & $\begin{array}{l}\text { 令 } \\
\stackrel{d}{d} \\
\stackrel{D}{1}\end{array}$ & 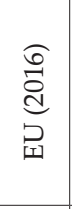 & 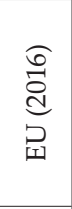 & 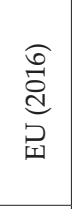 & 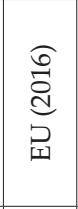 & \\
\hline 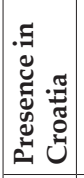 & 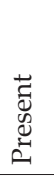 & 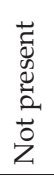 & 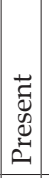 & 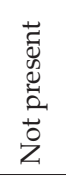 & 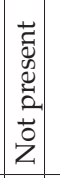 & $\begin{array}{l}\overrightarrow{D^{\prime}} \\
0 \\
0 \\
\vec{D} \\
\overrightarrow{0} \\
z\end{array}$ & \begin{tabular}{|l|}
$\vec{z}$ \\
0 \\
0 \\
$\vdots$ \\
0 \\
0 \\
0 \\
$z$ \\
\end{tabular} & 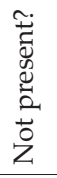 & 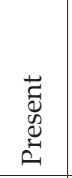 & $\begin{array}{l}\overrightarrow{0} \\
0 \\
0 \\
0 \\
0 \\
0 \\
Z\end{array}$ & $\mid$\begin{tabular}{|c|}
$\overrightarrow{0}$ \\
0 \\
0 \\
0 \\
0 \\
0 \\
$z$ \\
$z$
\end{tabular} & 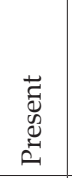 & 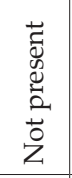 & 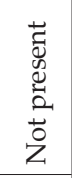 & $\begin{array}{l}\vec{D} \\
\vec{D}\end{array}$ & $\begin{array}{l}\infty \\
\stackrel{\infty}{2} \\
\overrightarrow{2}\end{array}$ & 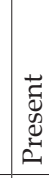 \\
\hline . & 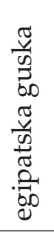 & 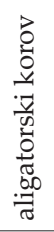 & 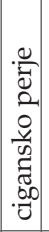 & 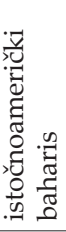 & \begin{tabular}{|c|}
$\tilde{z}$ \\
$\tilde{\Xi}$ \\
$\tilde{0}$ \\
$\tilde{0}$ \\
$\tilde{\tilde{\Xi}}$ \\
\end{tabular} & 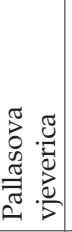 & 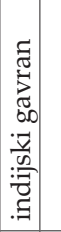 & 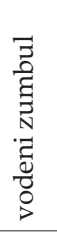 & 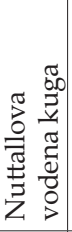 & 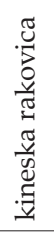 & 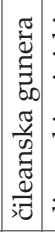 & 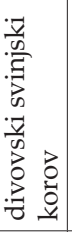 & 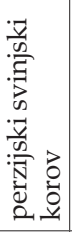 & 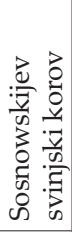 & 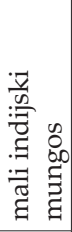 & 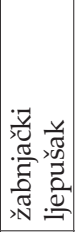 & $\begin{array}{l}4 \\
2 \\
2\end{array}$ \\
\hline
\end{tabular}

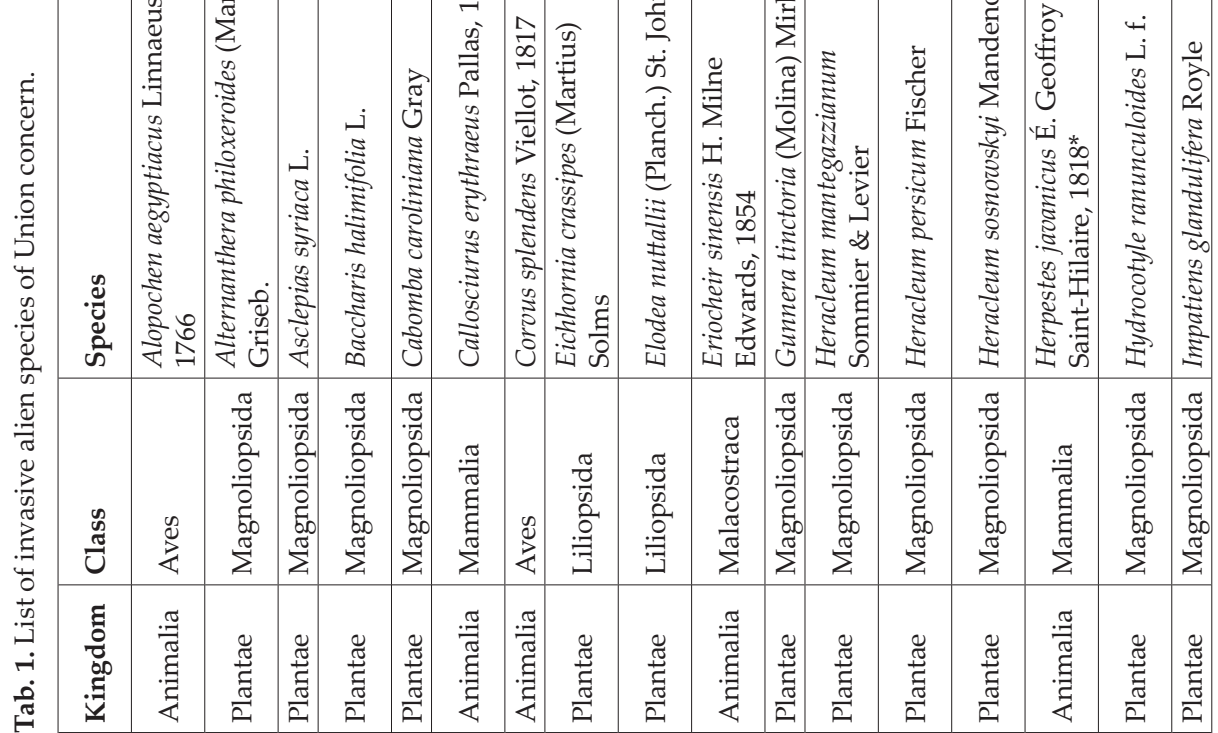




\begin{tabular}{|c|c|c|c|c|c|c|c|c|c|c|c|c|c|c|c|c|c|c|}
\hline 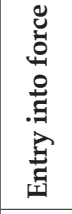 & 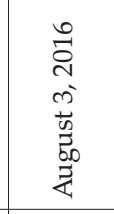 & 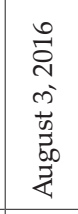 & 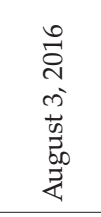 & 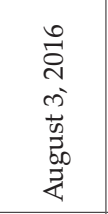 & 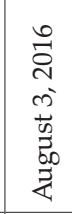 & 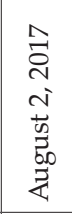 & 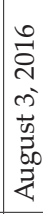 & 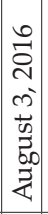 & 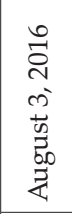 & 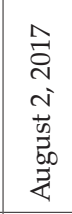 & 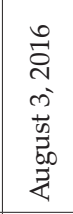 & 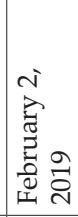 & 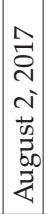 & 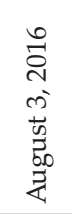 & 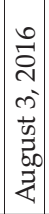 & 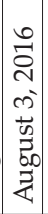 & $\mid \begin{array}{c}0 \\
0 \\
2 \\
2 \\
0 \\
0 \\
0 \\
0 \\
0 \\
0 \\
z \\
z\end{array}$ & 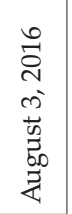 \\
\hline 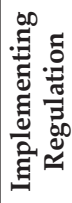 & 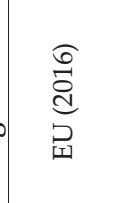 & 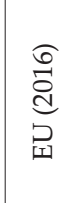 & $\begin{array}{l}0 \\
\stackrel{0}{0} \\
\stackrel{0}{0} \\
\stackrel{0}{1}\end{array}$ & 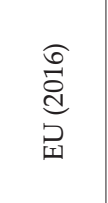 & 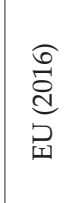 & 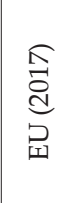 & 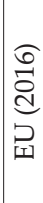 & 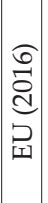 & 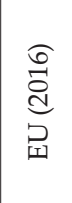 & 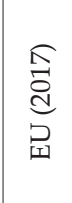 & 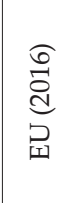 & 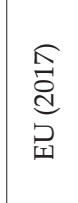 & 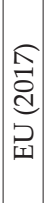 & 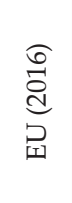 & 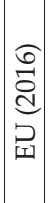 & 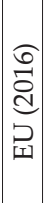 & $\mid \begin{array}{c}0 \\
0 \\
0 \\
d \\
0 \\
p \\
1\end{array}$ & 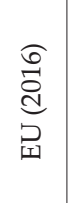 \\
\hline 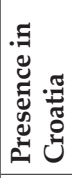 & $\begin{array}{l}\overrightarrow{0} \\
0 \\
0 \\
0 \\
0 \\
0 \\
z\end{array}$ & $\begin{array}{l}\tilde{D} \\
0 \\
0 \\
0 \\
0 \\
0 \\
Z\end{array}$ & 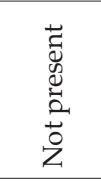 & 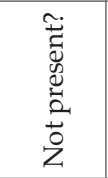 & 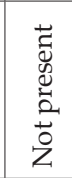 & 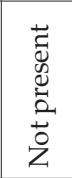 & $\begin{array}{l}\overrightarrow{0} \\
0 \\
0 \\
0 \\
0 \\
0 \\
0\end{array}$ & 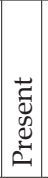 & 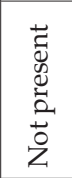 & 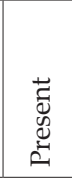 & 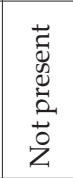 & 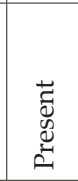 & 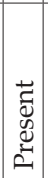 & 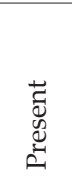 & 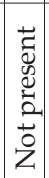 & 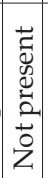 & 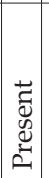 & $\begin{array}{l}\overrightarrow{D_{0}} \\
\stackrel{0}{0} \\
\stackrel{0}{0} \\
0 \\
z\end{array}$ \\
\hline 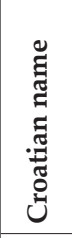 & 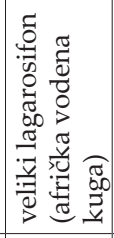 & 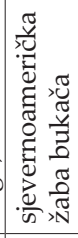 & 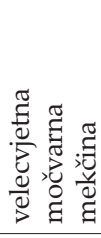 & 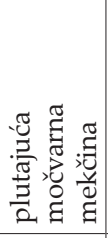 & 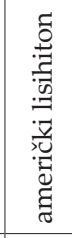 & 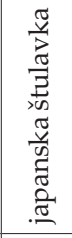 & 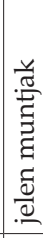 & 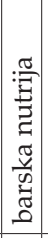 & 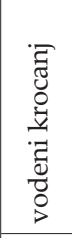 & 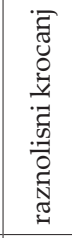 & 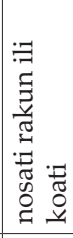 & 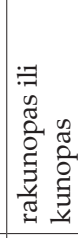 & 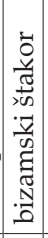 & 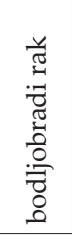 & 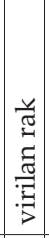 & 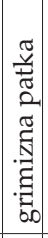 & 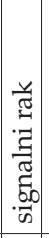 & 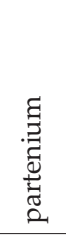 \\
\hline 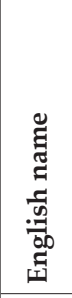 & 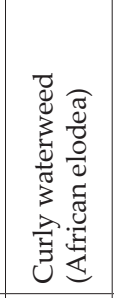 & 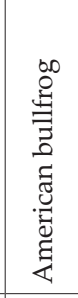 & 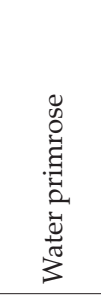 & 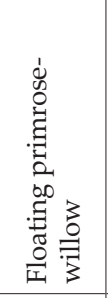 & 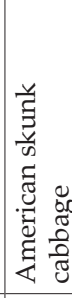 & 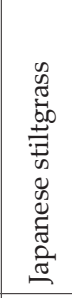 & 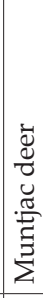 & 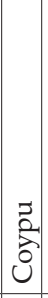 & 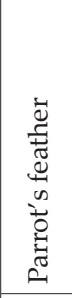 & 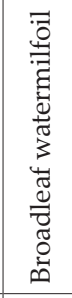 & 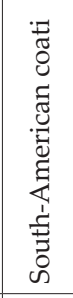 & 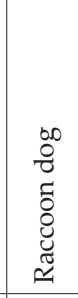 & 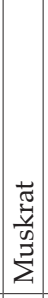 & 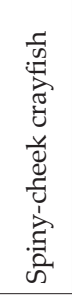 & 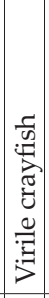 & 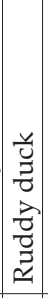 & 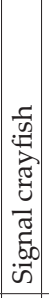 & 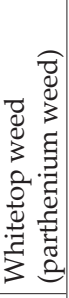 \\
\hline $\begin{array}{l}\text { क्ष } \\
\text { के } \\
\text { के }\end{array}$ & 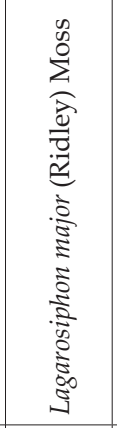 & 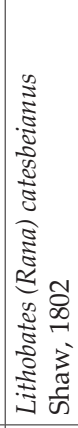 & 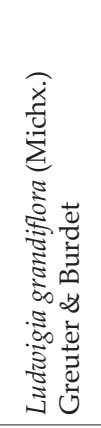 & 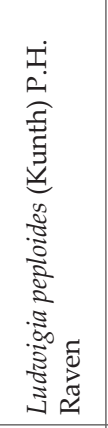 & 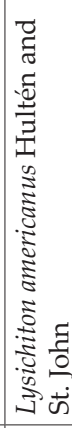 & 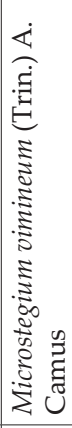 & 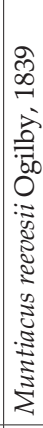 & 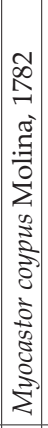 & 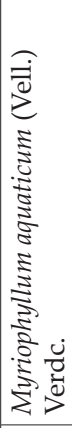 & 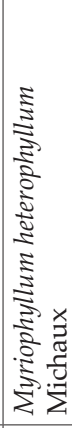 & 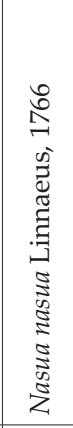 & 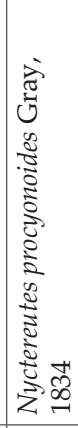 & 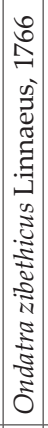 & 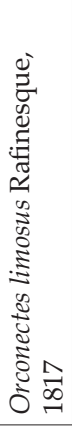 & 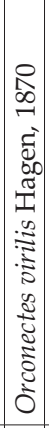 & 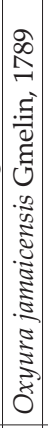 & 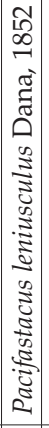 & 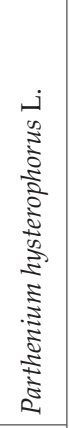 \\
\hline 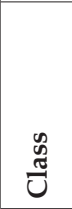 & $\begin{array}{l}\frac{\pi}{0} \\
\frac{0}{n} \\
\frac{2}{9} \\
: 7\end{array}$ & $\begin{array}{l}\frac{\pi}{3} \\
\frac{\pi}{3} \\
\frac{2}{2} \\
\frac{1}{2}\end{array}$ & 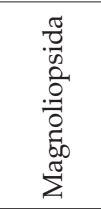 & 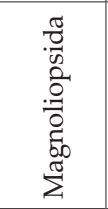 & $\begin{array}{l}\frac{\pi}{0} \\
\frac{0}{0} \\
\stackrel{0}{0} \\
:=7\end{array}$ & $\begin{array}{l}\frac{\pi}{0} \\
\frac{0}{0} \\
\frac{2}{0} \\
: 7 \\
:=1\end{array}$ & 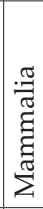 & 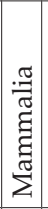 & 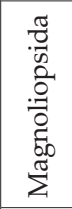 & 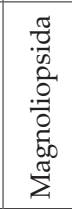 & 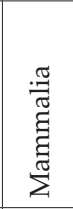 & 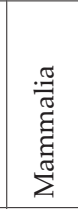 & 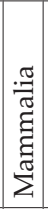 & 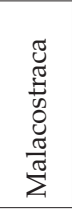 & 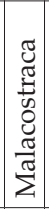 & 足 & 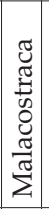 & 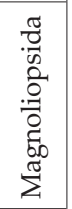 \\
\hline 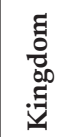 & $\underset{\mathbb{J}}{\mathbb{\Xi}}$ & 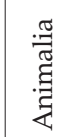 & $\underset{\underset{\mathbb{Z}}{\tilde{I}}}{\mathbb{\Xi}}$ & $\begin{array}{l}\frac{\tilde{\Xi}}{\tilde{\Xi}} \\
\frac{\pi}{\sim}\end{array}$ & $\begin{array}{l}\frac{\tilde{J}}{\Xi} \\
\frac{\pi}{\sigma}\end{array}$ & $\frac{\mathbb{\Xi}}{\underset{\tilde{J}}{\tilde{U}}}$ & 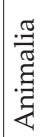 & 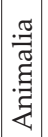 & 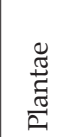 & $\frac{\underset{\tilde{J}}{\tilde{U}}}{\frac{\vec{U}}{\tilde{E}}}$ & $\begin{array}{l}\text { 氶 } \\
\text { 节 } \\
\text { 芸 }\end{array}$ & $\begin{array}{l}\text { 苂 } \\
\text { 节 }\end{array}$ & 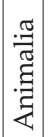 & 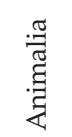 & 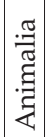 & 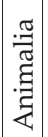 & 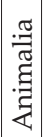 & 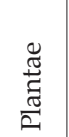 \\
\hline
\end{tabular}




\begin{tabular}{|c|c|c|c|c|c|c|c|c|c|c|c|c|c|c|}
\hline 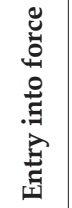 & 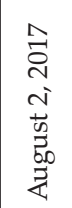 & 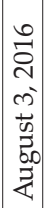 & 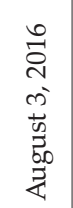 & 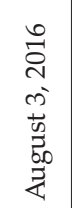 & 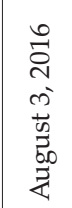 & 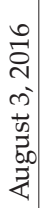 & 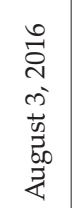 & 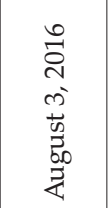 & 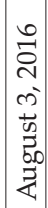 & 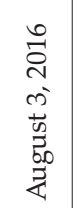 & 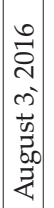 & 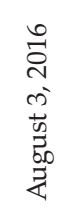 & 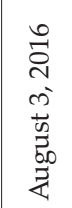 & 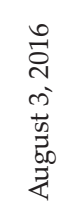 \\
\hline 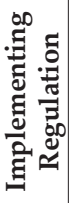 & 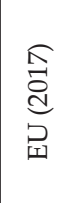 & 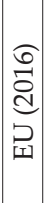 & 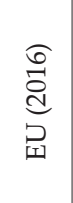 & 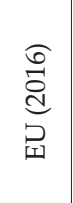 & 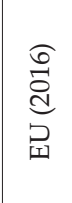 & 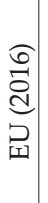 & 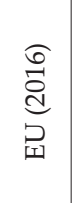 & 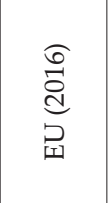 & 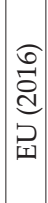 & 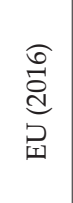 & 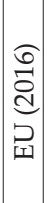 & 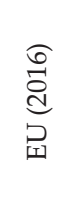 & 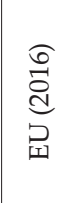 & 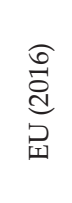 \\
\hline 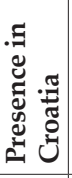 & 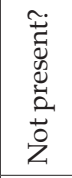 & 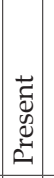 & 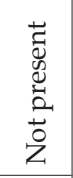 & 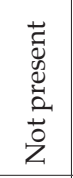 & 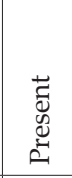 & 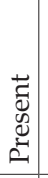 & 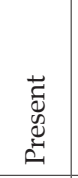 & 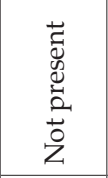 & 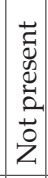 & $\begin{array}{l}\overrightarrow{\tilde{U}} \\
\stackrel{0}{0} \\
\stackrel{0}{2} \\
\overrightarrow{0} \\
z\end{array}$ & 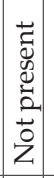 & $\begin{array}{l}\overrightarrow{\tilde{U}} \\
\stackrel{0}{0} \\
\tilde{0} \\
\overrightarrow{0} \\
z\end{array}$ & 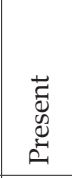 & 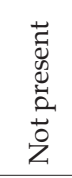 \\
\hline 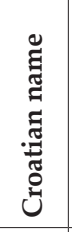 & 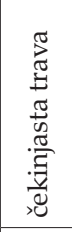 & 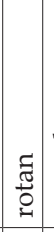 & 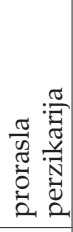 & 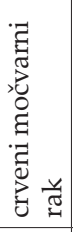 & 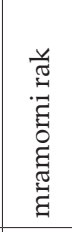 & 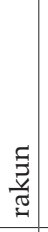 & 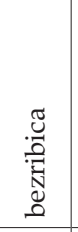 & 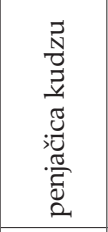 & 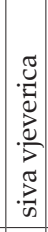 & 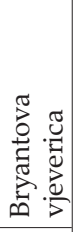 & 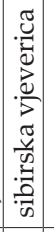 & 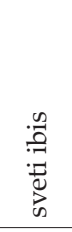 & 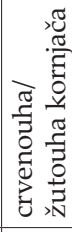 & 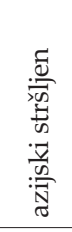 \\
\hline 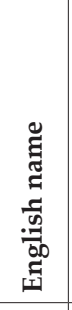 & 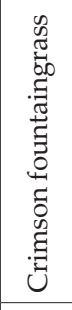 & 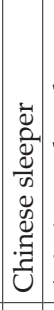 & 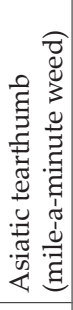 & 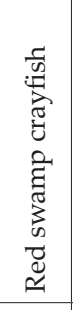 & 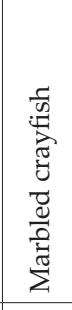 & $\begin{array}{l}0 \\
0 \\
0 \\
\tilde{\Xi} \\
\simeq \\
\end{array}$ & 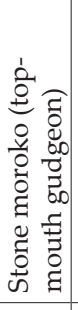 & 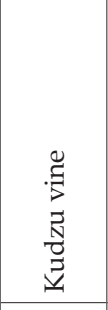 & 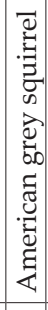 & 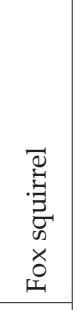 & 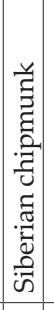 & 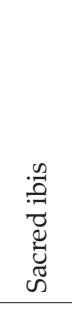 & 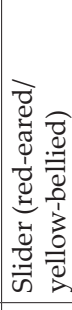 & 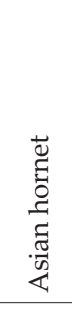 \\
\hline $\begin{array}{l}\text { के } \\
\text { के } \\
\text { के }\end{array}$ & 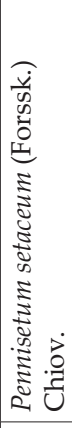 & 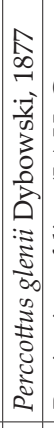 & 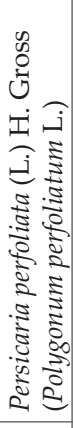 & 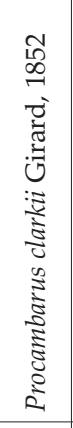 & 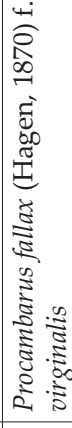 & 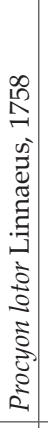 & 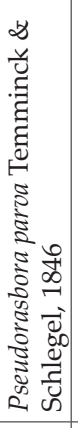 & 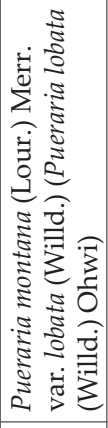 & 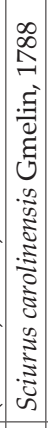 & 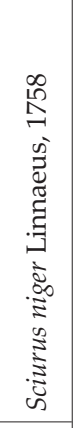 & 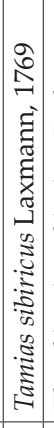 & 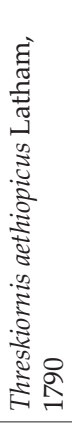 & 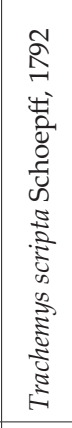 & 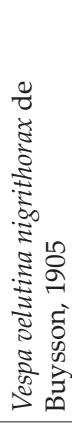 \\
\hline $\begin{array}{l}\text { o } \\
\text { Uే }\end{array}$ & 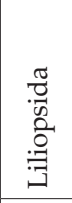 & 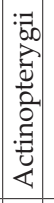 & 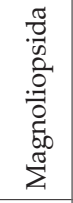 & 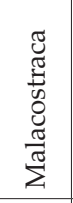 & 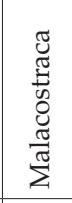 & 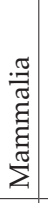 & 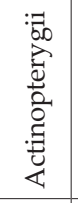 & $\begin{array}{l}\frac{\pi}{0} \\
\frac{0}{n} \\
\tilde{2} \\
.7 \\
0 \\
0 \\
5 \\
\tilde{\sigma} \\
\Sigma\end{array}$ & 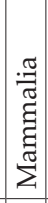 & 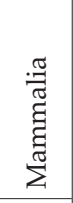 & 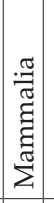 & $\sum^{\circ}$ & 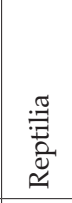 & 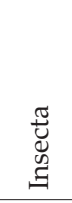 \\
\hline 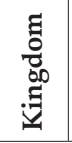 & $\begin{array}{l}\frac{\mathbb{\Xi}}{\mathbb{\Xi}} \\
\frac{\pi}{\pi}\end{array}$ & 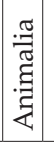 & $\begin{array}{l}\frac{\tilde{\Xi}}{\stackrel{\Xi}{*}} \\
\frac{\pi}{\pi}\end{array}$ & 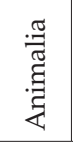 & $\begin{array}{l}\text { 永 } \\
\text { 昙 } \\
\end{array}$ & 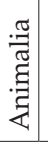 & 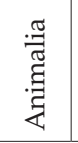 & $\begin{array}{l}\frac{\tilde{J}}{\tilde{\Xi}} \\
\frac{\pi}{\tilde{N}}\end{array}$ & 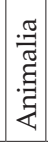 & 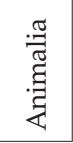 & 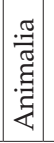 & 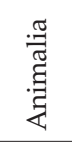 & 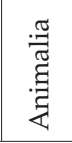 & 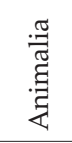 \\
\hline
\end{tabular}



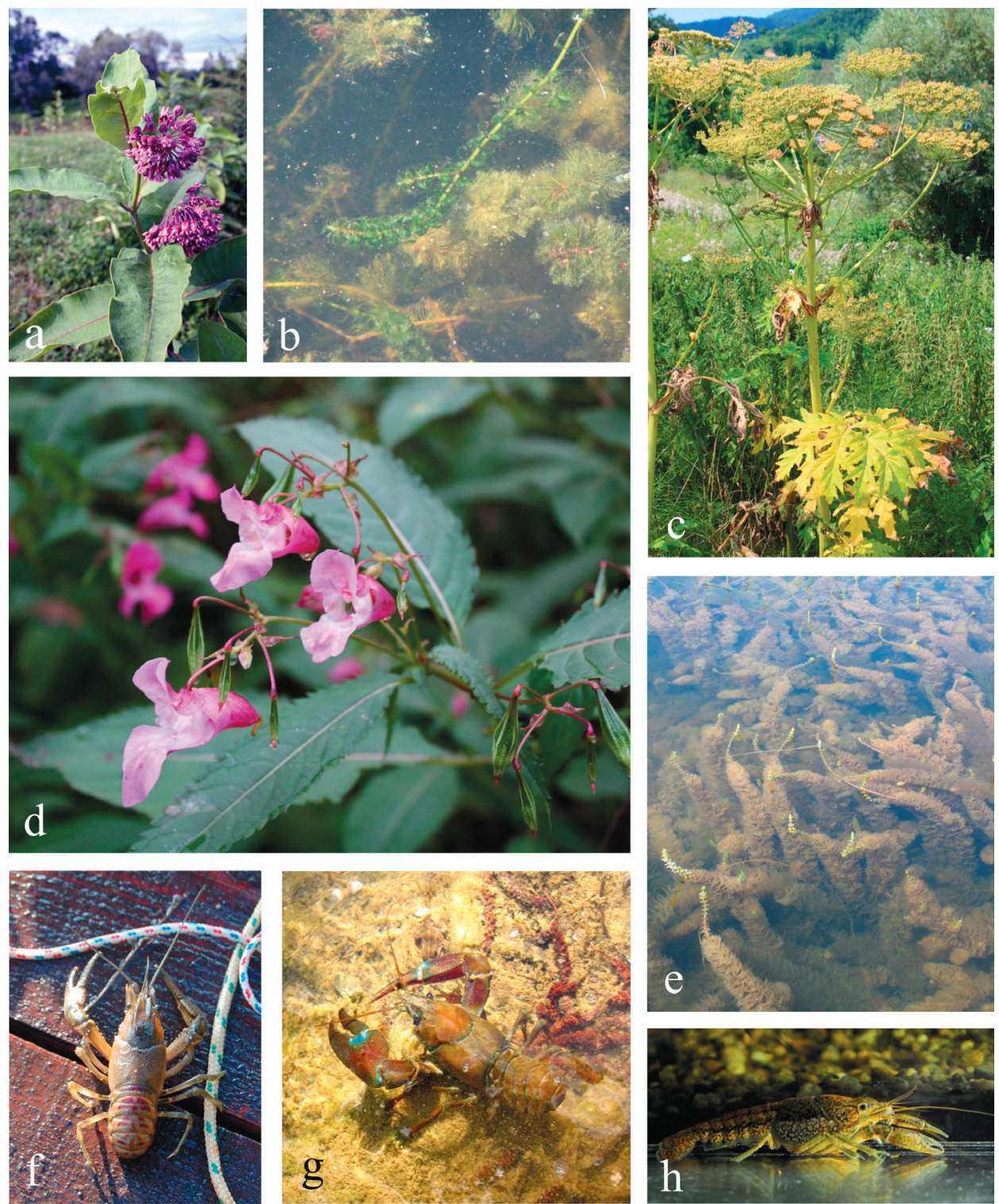

Fig. 1. Species of Union concern present in Croatia: a) Asclepias syriaca L. (photo by I. Boršić); b) Elodea nuttallii (Planch.) St. John (photo by A. Kočić); c) Heracleum mantegazzianum Sommier \& Levier (photo by I. Boršić); d) Impatiens glandulifera Royle (photo by I. Boršić); e) Myriophyllum heterophyllum Michaux (photo by I. Boršić); f) Orconectes limosus (Rafinesque, 1817) (photo by S. Hudina); g) Pacifastacus leniusculus (Dana, 1852) (photo by L. Katušić); h) Procambarus fallax (Hagen, 1870) f. virginalis (photo by M. Samardžić). 

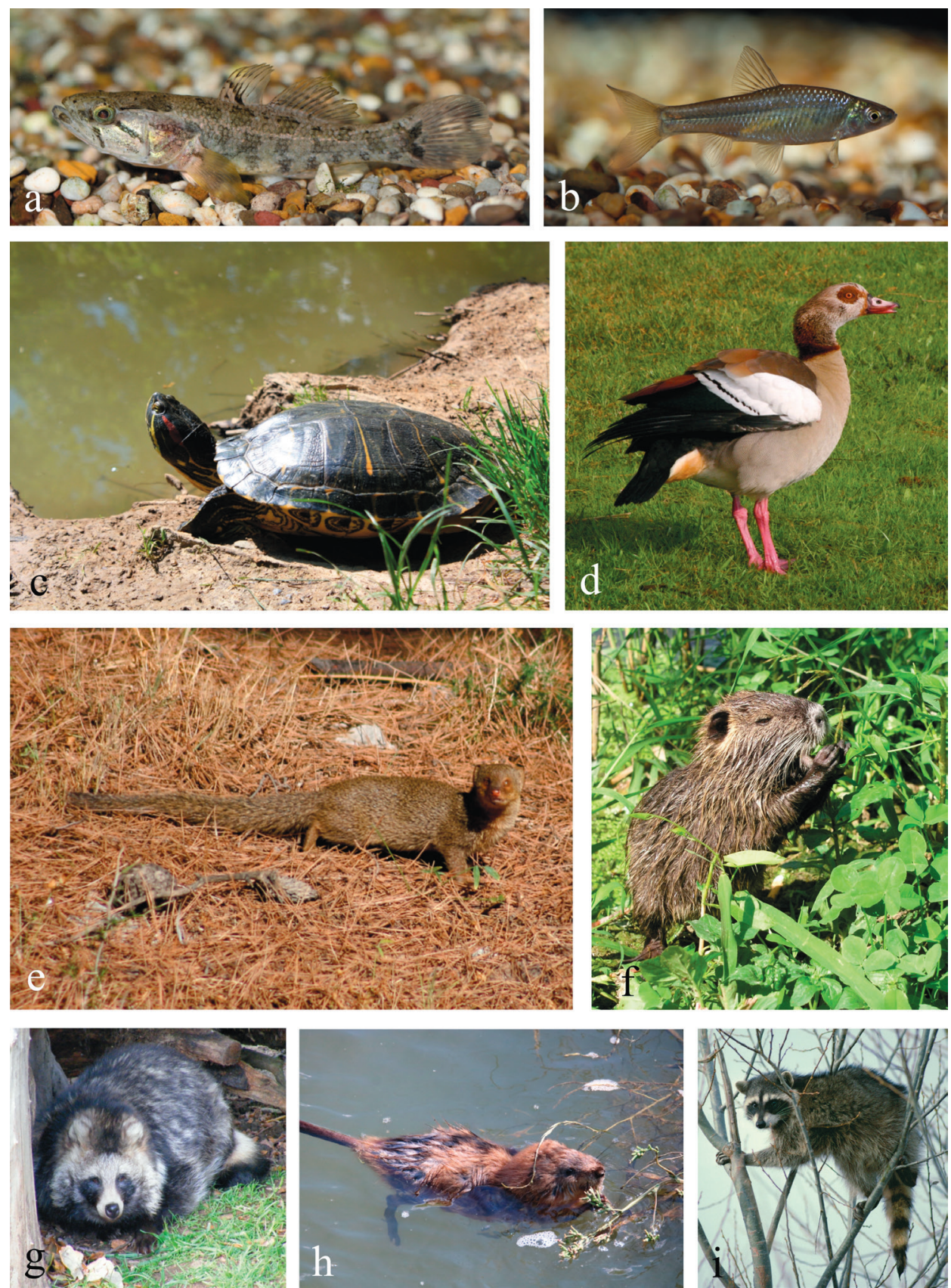

Fig. 2. Species of Union concern present in Croatia: a) Pseudorasbora parva Dybowski, 1877 (photo by P. Mustafić); b) Perccottus glenii (Temminck \& Schlegel, 1846) (photo by P. Mustafić); c) Trachemys scripta (Schoepff, 1792) (photo by I. Boršić); d) Alopochen aegyptiacus (Linnaeus, 1766) (source: Wikimedia Commons); e) Small Indian mongoose (photo by M. Franković, NP Mljet archives); f) Myocastor coypus (Molina, 1782) (photo by I. Boršić); g) Nyctereutes procyonoides (Gray, 1834) (photo by Marie Hale); h) Ondatra zibethicus (Linnaeus, 1766) (photo by I. Boršić); i) Procyon lotor (Linnaeus, 1758) (photo by David Menke, U.S. Fish and Wildlife Service). 
The native range of $A$. syriaca is eastern North America. It was introduced to Europe in $17^{\text {th }}$ century as an ornamental plant (BAgI, 2008; Petrova et al., 2013). In Europe it is naturalized in Austria, Bulgaria, Croatia, the Czech Republic, Denmark, France, Germany, Hungary, Italy, Lithuania, the Netherlands, Poland, Romania, Slovakia, Slovenia, and Spain; and it is considered invasive in most of those countries (NOBANIS, 2017; DAISIE, 2018). Regarding its impact, it is an aggressive and persistent agricultural weed (CABI, 2018b) with negative allelopathic effects (BAGI, 2008). By infesting arable land and by distracting pollinators it causes crop losses (BAGI, 2008). It is a host for the cucumber mosaic virus (CMV) and for the pest insect Frankliniella occidentalis which is a vector of tomato spotted wilt virus (TSWV; BAGI, 2008). It contains glycosides that are poisonous to livestock (CABI, 2018b). It has negative physical effects (shade and space occupation) on native species (BAGI, 2008) and changes the composition of native communities (Nikolić et al., 2014).

Supposedly it was introduced to Croatia as an ornamental plant which subsequently escaped from cultivation and started spreading. The first records in Croatia date from the second half of the $19^{\text {th }}$ century (Nikolić et al., 2014). It is a common, widely spread species, distributed primarily in northwestern and eastern Croatia, with rare records elsewhere (e. g. island of Lošinj, Murter, Brela) (cf. NiкоLıć, 2018). Although it is considered an invasive alien plant species (Boršić et al., 2008), it is still used as an ornamental and melliferous plant. Occupied habitats include anthropogenically influenced habitats, along the roads and railways, on river embankments, on pastures and in open forest areas (Nikolić et al., 2014).

Elodea nuttallii (Planch.) St. John - Nuttall's waterweed (Croatian: Nuttallova vodena kuga); Figs. 1b, 4

The species Elodea nuttallii is a rooted, submerged freshwater perennial macrophyte. It has thin, filiform roots at the stem nodes. Stems are long, slender and often branched. Leaves are linear or linear-lanceolate, gradually tapering towards an acute apex, lower are opposite, while upper are in whorls of 3(4). It is a dioecious plant with unisexual, white-violet flowers about $4 \mathrm{~mm}$ wide. The male flower while still in bud breaks free and rises to the surface, where it opens and releases pollen, while the female flower also floats on the water surface, but remains connected to the plant. Fruit is a capsule, with 1-5 seeds. It flowers from June to September (DANDy, 1980; Preston \& Croft, 1997; Josefsson, 2011; Petrova et al., 2013).

The species is native to temperate North America. In Europe it is known from the beginning of $20^{\text {th }}$ century - it was recorded in Great Britain in 1914 (firstly as Hydrilla verticillata (L.f.) Royle), followed by a record from Belgium in 1939. It was most likely introduced as an ornamental plant which was then accidentally released from aquariums (Josefsson, 2011). Today in Europe it is naturalized in Austria, Belgium, Bulgaria, Denmark, France, Germany, Great Britain, Hungary, Ireland, Italy, Luxembourg, Netherlands, Norway, Romania, Serbia, Slovakia, Slovenia, Sweden, and Switzerland (Kočıć et al., 2014; NOBANIS, 2017; DAISIE, 2018). In central Europe both male and female plants are present (KRAUSCH, 1996), but it mainly reproduces vegetatively and spreads by vegetative fragments (Preston \& CROFT, 1997). It grows in eutrophic, oligo- and mesoprobic waters, where it can form dense monospecific stands, changing the chemical composition of water as well as the composition of species, e.g. displacement of other plant species (because of competition for nutrients and shading) or impact on 


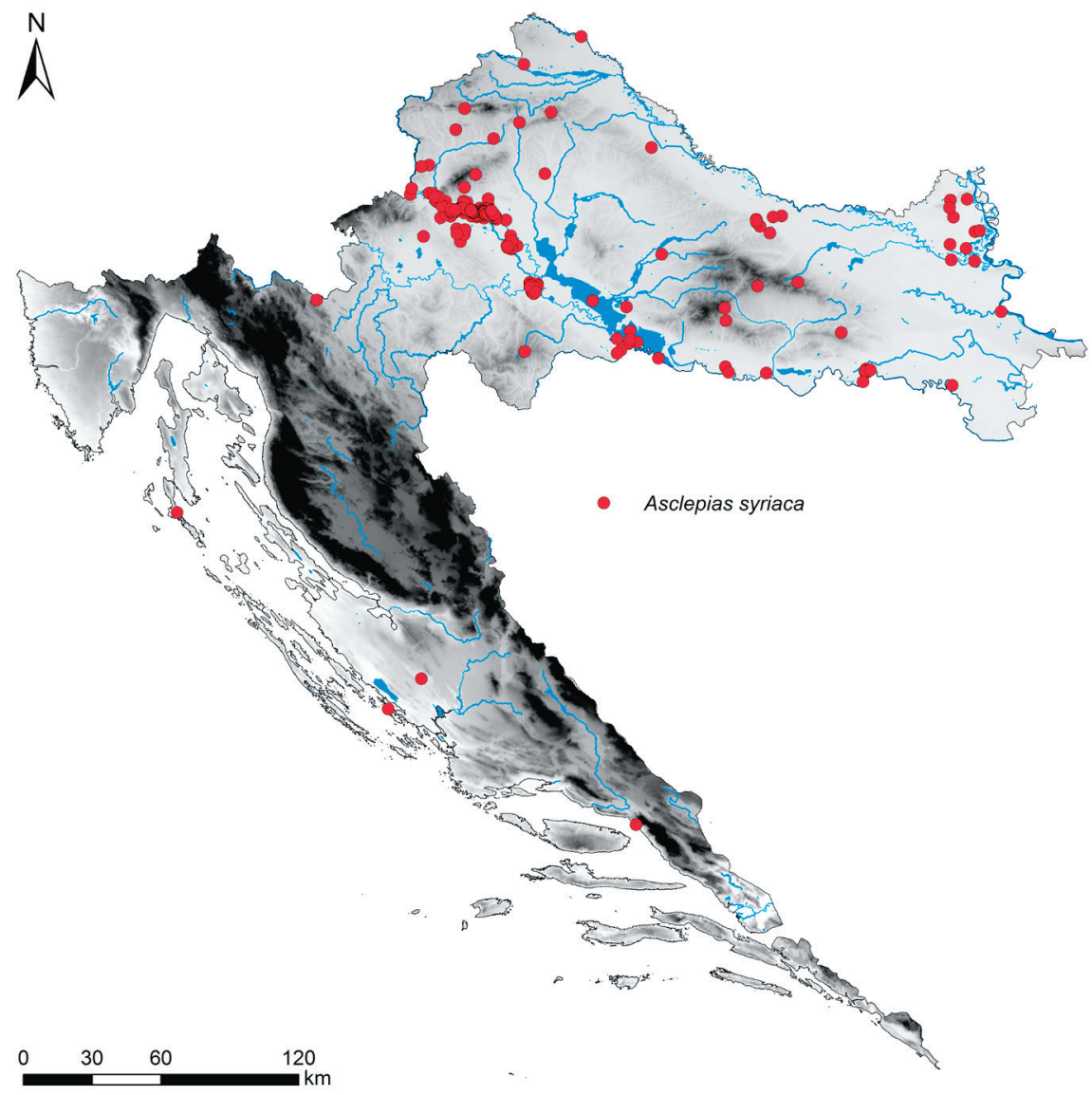

Fig. 3. Distribution of Asclepias syriaca L. in Croatia.

animal species. It can reduce water flow in drainage canals and negatively impact water resources and recreational activities (JosEFsson, 2011; CABI, 2018c).

It was found for the first time in Croatia in 2006, in Kopački rit floodplain (the mouth of the River Drava into the Danube). Subsequently, the species became established in Baranja, where it spread along the network of drainage canals. It was most likely introduced from Hungary along the Danube River (Kočıć et al., 2014). It was also recorded in the Drava River (Vidović, 2013; 2015), where it was apparently introduced from Slovenia (Kočić et al., 2014).

Heracleum mantegazzianum Sommier \& Levier - giant hogweed (Croatian: divovski svinjski korov); Figs 1c, 4

The species Heracleum mantegazzianum is a monocarpic perennial herb that persists several years in a rosette stage. It can grow up to $5 \mathrm{~m}$ high. It has ridged stem with purple blotches and pustulate bristles. Leaves are up to $300 \mathrm{~cm}$ long, alternately, ternately or pinnately lobed and coarsely toothed. Flowers are white or rarely pinkish. The inflorescence is compound umbel, with one terminal and up to eight satellite umbels. Fruits 
are flattened and elliptical, narrowly winged, splitting into two mericarps, each with 3-5 strongly swollen vittae. Seeds are efficiently dispersed by water, wind and human-related factors. It flowers from June to August (BRummitт, 1968; Boršıć et al., 2015; CABI, 2018d).

The species is native to the Western Greater Caucasus (Russia, Georgia). It was noted in Estonia in 1814, and it was reported as naturalized for the first time in 1828, in Cambridgeshire, from the plants introduced at the beginning of the $19^{\text {th }}$ century to the Kew Botanic Gardens in London (KLINGENSTEIN, 2007). Later it became widely distributed due to its ornamental usage, usage as fodder plant and food plant for honeybees, and its subsequent spontaneous spread (EPPO, 2009). Today in Europe it is naturalized in numerous countries (MASLO, 2010; NOBANIS, 2017; DAISIE, 2018), and in many of them it is considered invasive (NOBANIS, 2017). Besides disturbed habitats, which it invades in high densities, forming monospecific stands, it also grows in semi-natural and natural habitats. Because of its size it has significant negative physical effects (shade and space occupation) in the invaded habitats, where it can outcompete and replace native species. Furthermore, it might hybridise with native species, especially Heracleum sphondylium L. (STEWART \& GRACE, 1984). It has a strong negative impact on human health. It produces phototoxic sap which contains photosensitizing furanocoumarins. Contact with the plant and exposure to the sunlight causes severe skin burns (KLINGENSTEIN, 2007; CABI, 2018d).

In Croatia it was first recorded in 2009 near Žabnik (Međimurje County; STUNKović, 2009), where it has not been found since. In 2014 it was recorded in Gornja Šemnica (Radoboj, Krapina-Zagorje County), where it grows in the ruderal vegetation of the order Onopordetalia acanthii Br.-Bl. et R. Tx. ex Klika et Hadač 1944 mixed with ruderal vegetation of the class Galio-Urticetea Passarge ex Kopecký 1969 and in hygrophilous herbaceous vegetation of the alliance Calthion R. Tx. 1936 (Boršić et al., 2015). Although it is an ornamental plant that escapes from gardens, unintentional introduction was presumed (Boršić et al., 2015).

\section{Impatiens glandulifera Royle - Himalayan balsam, policeman's helmet (Croatian: žljezdasti nedirak); Figs. 1d, 5}

The species Impatiens glandulifera is an annual herb up to $250 \mathrm{~cm}$ tall. It has a thick, glabrous and simple stem, with a $15 \mathrm{~cm}$ deep primary root and adventive roots that develop at the lower nodes of the stem. Leaves are opposite or in whorls of three in the upper part, lanceolate to elliptic, cuneate and glandular at the base, and with serrate margin. Flowers are showy, strongly zygomorphic, pink to purple or rarely white. The calyx is made of three sepals, two lateral sepals are small, triangular and mostly green, while the lowest sepal forms a sac that abruptly ends in a straight spur. Corolla is made of five petals, the longest upper petal and four lower petals which are fused in two lateral pairs, except their apical parts. The inflorescences are axillary racemes of up to 12 flowers. Fruit is a glabrous, clavate capsule with 5 valves, which opens explosively and disperses seeds. It flowers from June to August (Walters, 1968; HejdA, 2009; Petrova et al., 2013; Nikolić, 2014; CABI, 2018e).

It originates from the Himalayas, northern Pakistan, northern India, and Nepal. In Europe it was first introduced as an ornamental garden plant to the United Kingdom in 1839. Today it is naturalized in the majority of European countries (HeJdA, 2009; Helmisaari, 2010; Petrova et al., 2013; Maslo, 2016; CABI, 2018e) and in many of them it is considered invasive (NOBANIS, 2017). It predominantly grows along river banks, but also along railways, in urban areas, in landfills, and in natural habitats such as 


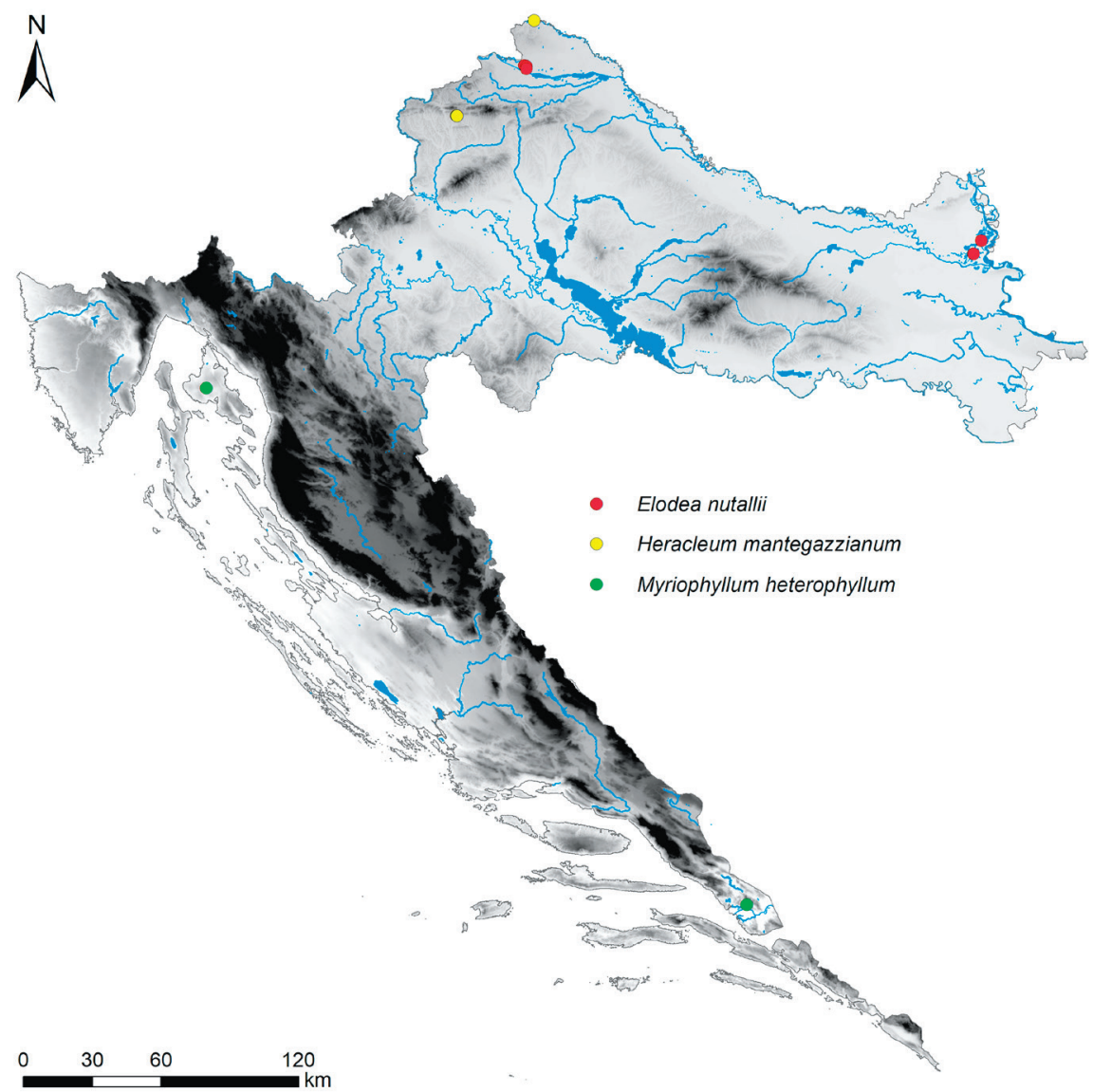

Fig. 4. Distribution of Elodea nuttallii (Planch.) St. John, Heracleum mantegazzianum Sommier \& Levier, and Myriophyllum heterophyllum Michaux in Croatia.

forests, forest clearings, and wet meadows. Because it is an annual plant, it does not have as strong an impact on native species as some perennial invasive plants. However, it causes changes in the cover and composition of native species and can outcompete them by forming dense monospecific stands. It competes with native species for pollinators and causes changes of invertebrate communities. Furthermore, it can also impacts survival of forest seedlings (Cigić et al., 2003; HelmisaARI, 2010; CABI, 2018e).

In Croatia it was recorded for the first time in 1968 along the Sava River upstream of Zagreb where it spread from Slovenia (MARкоvić, 1970). Afterwards it was discovered in Skadar in Gorski Kotar (TRINAJSTIĆ, 1974). Its spread was well documented by Marković (1984), Lukač (1989), Pavletić (1993), Trinajstić \& Franjić (1994), Franjić et al. (1998) and PANDžA et al. (2001). It spreads along the watercourses, and from village gardens where it was used as ornamental plant (PAvLETIĆ, 1993). Today it is primarily distributed in the northwestern part of Croatia, with rare records in eastern Croatia and Pokuplje (Nikolić, 2018). It is considered an invasive alien plant species (Boršıć et al., 2008). It is a weedy-ruderal species which occurs in various vegetation types (MARKović 1984). It grows in ruderal vegetation (e.g. Ass. Urtico-Sambucetum ebuli Br.- 
Bl. (1936) 1952; Pavletić, 1993), on disposal sites, along the roads and railways. It also occupies natural habitats; it grows along rivers, in alluvial forests and on their edges, in the alliance Convolvulion sepium Oberd. 1949 (forming Ass. Impatienti-Solidaginetum M. Moor 1958; Trinajstić, 1995).

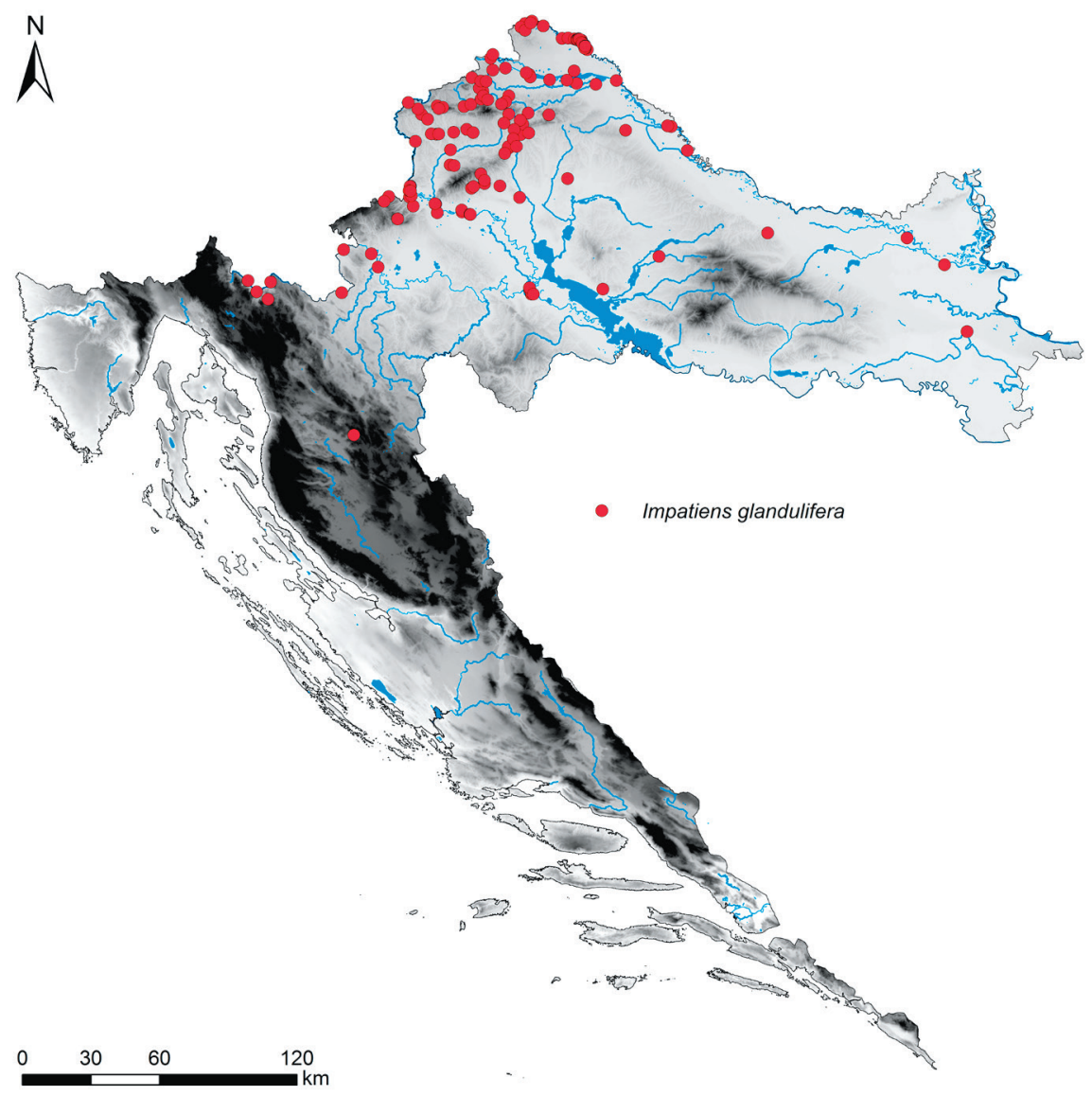

Fig. 5. Distribution of Impatiens glandulifera Royle in Croatia.

Myriophyllum heterophyllum Michaux - broadleaf watermilfoil, changeleaf parrotfeather (Croatian: raznolisni krocanj); Figs. 1e, 4

The species Myriophyllum heterophyllum is a rhizomatous, submerged freshwater perennial macrophyte. Stems are up to $100 \mathrm{~cm}$ long. Leaves have different forms. Submerged leaves are feather-like and pinnate, with 4-10 pinnae. Emergent leaves are either pinnately dissected (terrestrial form) or entire toothed (an emergent leaf form, on the flowering stem). Flowers are produced only on the emergent part of the stem which can often be exposed 10-15 cm above the water surface. Spike with solitary flowers that are borne in the axils of lanceolate bracts. Flowers are usually hermaphrodite, or female flowers are occasionally at the base of the inflorescence and male above. There are four petals and no sepals. However, flowering is rarely observed. Fruit separates longitu- 
dinally into four subglobose, finely tuberculate nutlets. Each nutlet is beaked and has two ridges on the outer face. It flowers from June to July (CooK, 1968; KRAusch, 1996; EPPO, 2016).

The native range of $M$. heterophyllum is eastern North America. In Europe it was first discovered in 1941 in Great Britain, in a canal between Halifax and Salterhebble, where it was naturalized until the drainage of the canal in 1947 or 1948 (PRESTON \& CROFT, 1997). Today it is naturalized in Austria, Belgium, France, Germany, Hungary, Luxembourg, the Netherlands, Spain, and Switzerland (EPPO, 2015; EPPO, 2016; LukÁcs et al., 2016; DAISIE, 2018; NOBANIS, 2018). It is common in the aquarium plant trade, where it can be found under a variety of names. It grows in freshwater habitats: slowly moving rivers, canals, lakes, ponds, reservoirs, and wetlands. By forming dense mats the species can reduce oxygen availability and sunlight, and can outcompete other aquatic plants. Furthermore, it has negative effects on human activities in water by reducing water availability and flow (EPPO, 2016; CABI, 2018f).

The first record of $M$. heterophyllum for Croatia was from the small Lake Ponikve on Krk island in 2000, published nine years later (STARMüHLER, 2009). However, this finding passed unnoticed both in Croatia and on the international level (EPPO, 2016). The second and the most recent finding was from Lake Desne in the Neretva River Delta in 2016, where the species was growing within the vegetation of rooted leaf-floating macrophytes of the alliance Nymphaeion albae Oberd. 1957 and order Potamogetonetalia Koch 1926 (JAsprica et al., 2017). The mode of its introduction to Croatia is not known.

\section{Freshwater crayfish}

Three alien freshwater crayfish of Union concern are currently known for Croatia: Procambarus fallax (Hagen, 1870) f. virginalis and Orconectes limosus (Rafinesque, 1817) from family Cambaridae, and Pacifastacus leniusculus (Dana, 1852) belonging to family Astacidae, all within the order Decapoda. They originate from North America and are naturalized widely across Europe, including Croatia.

\section{Orconectes limosus (Rafinesque, 1817) - spiny-cheek crayfish (Croatian: bodljo- bradi rak); Figs. 1f, 6}

According to an updated classification system of the freshwater crayfishes (Decapoda: Astacidae) of the world (Crandal \& De Grave, 2017) Faxonius limosus is the valid taxonomic name for Orconectes limosus (the name currently listed on the Union list). It is a small to medium sized crayfish, the largest specimens reaching just over 10 $\mathrm{cm}$. The body is usually dark brown with dorsal part of abdomen striped with brownred transversal bands. The tips of the claws are characteristically orange, bordered by a dark band. The carapace is smooth, with distinct sharp spines laterally, in front and right behind of the cervical groove. It is adapted to a wide variety of environmental conditions, including brackish waters, organic and inorganic pollution, but it predominantly occurs in larger, warmer, slow-flowing or lentic waters (JAszczoŁT \& SzANIAWSKA, 2011; AleKHNOvich \& BǓ̌ič, 2017 and references therein).

It was the first alien crayfish intentionally introduced into Europe. It was brought to Poland from the USA in 1890s to replace the native European noble crayfish Astacus astacus (Linnaeus, 1758) stocks that were devastated by the crayfish plague. After introduction it had spread unaidedly through rivers and canals. It was also unintentionally translocated by human activity when used as fish bait or food, or transported with 
the fish stocks (Holdich \& BLACK, 2007). Additionally, deliberate stocking and translocation by boat traffic are possible pathways of introductions (PUKY \& SCHÁD, 2006). It is present in at least 24 European countries, with the most recent records from Bulgaria in 2015 and Slovenia in 2017 (Kouba et al., 2014; Trichкova et al., 2015; Govedič, 2017).

This is a fast-growing and highly fertile species, with the capacity for rapid dispersal (Maguire \& KLOBuČar, 2003; KozAK et al., 2007). For those reasons they often occur in higher densities than native crayfish and drastically affect their populations due to competition for food, and as a crayfish plague carriers (VogT, 1999). They can also negatively affect lower trophic levels in water ecosystems (VoJKOvsKa et al., 2014).

In 2003 O. limosus was detected in Kopački rit Nature Park in Croatia (Maguire \& KLOBUČAR, 2003). Since then it has significantly expanded its range in the Drava and Danube rivers in eastern Croatia (MAguire et al., 2011; MAguire et al., 2018). The presence of spiny-cheek crayfish in Croatia is probably the result of species dispersal from the Hungarian section of the Danube River. Although deliberate introduction for commercial purposes to and within northeastern Croatia cannot be excluded, they are unlikely (Hudina et al., 2009).

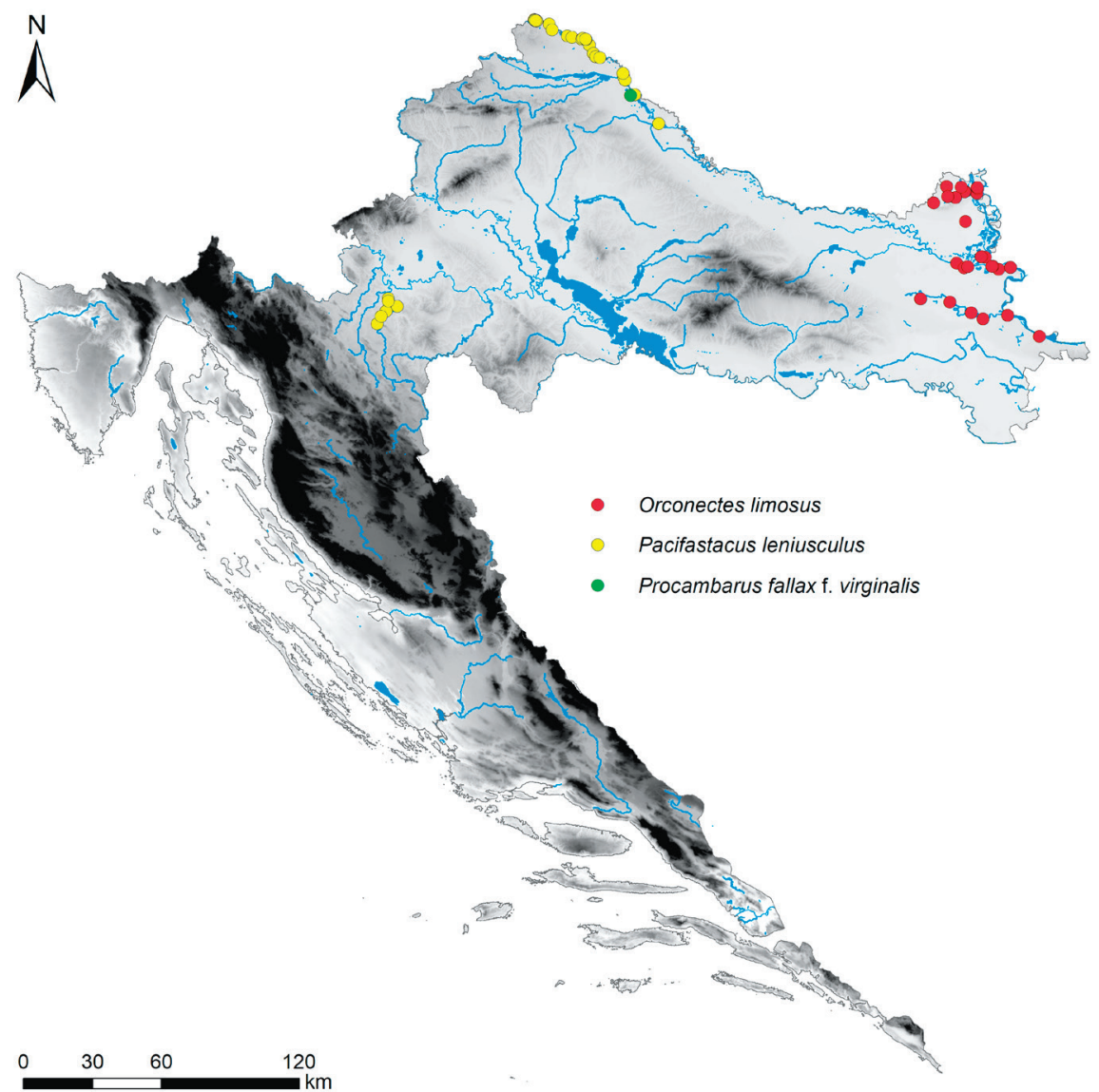

Fig. 6. Distribution of Orconectes limosus (Rafinesque, 1817), Pacifastacus leniusculus (Dana, 1852) and Procambarus fallax (Hagen, 1870) f. virginalis in Croatia. 


\section{Pacifastacus leniusculus (Dana, 1852) - signal crayfish (Croatian: signalni rak); Figs. 1g, 6}

The species Pacifastacus leniusculus has a similar appearance to that of the native noble crayfish (Astacus astacus), but it can be easily distinguished by its characteristic whiteturquoise patch on the upper side of the chelae, smoother chelae surface, and the absence of spines on the carapace shoulders, behind cervical groove (JoHNSEN \& TAUGBøL, 2010). The total body length of adult specimens is up to $15 \mathrm{~cm}$ for male and $13.4 \mathrm{~cm}$ for female (CAPURro et al., 2015). The signal crayfish is an omnivorous, opportunistic feeder (TurLEY et al., 2017). It inhabits a wide range of freshwater habitats (e.g. streams, rivers and lakes), but it can also survive in brackish water up to 20 ppt. salinity (LewIs, 2002).

The native range of signal crayfish is the western North American continent, from British Columbia in Canada to California in USA. However, it expanded its range within North America by human activities, and it was introduced to Japan and Europe (LewIS, 2002). Signal crayfish were brought to Sweden in 1960s to restore the important recreational and commercial crayfish fisheries, in the area where populations of native noble crayfish had been decimated by the crayfish plague. Since then, secondary introductions and natural dispersal of P. leniusculus in Europe continued (Johnsen \& TAUgBøL, 2010), despite restrictions prescribed by many governmental authorities, beginning from the late 1980s (Johnsen \& TAugbøL, 2010). To date P. leniusculus is the most widespread alien crayfish in Europe and its presence is confirmed in 29 countries (KoubA et al., 2014).

The signal crayfish owes much of its invasive success to its life history strategy, such as high fecundity, early maturation, and high aggressiveness (e.g. SöDERBÄCK, 1991; SöDERвÄсK, 1992; Usio et al., 2001). In Croatia it has the advantage in competition for food and shelter over native crayfish such as Astacus leptodactylus (e.g. Hudina et al., 2016), as well as over other alien crayfish such as Orconectes limosus (e.g. Hudina et al., 2011). Like other North American crayfish species, P. leniusculus can also transmit the crayfish plague (Vogt, 1999) to native crayfish species. The burrowing behaviour of the signal crayfish is shown to impact the streambed physical environment (GUAN, 1994; JoHnson et al., 2010).

In Croatia Pacifastacus leniusculus was recorded for the first time in the Mura River in 2008, as a result of downstream spread from Slovenia (MAGuire \& KLOBUČAR, 2008). Since then it expanded its range to the Drava River, with a potential to occupy water bodies in the entire Drava river catchment in northeastern Croatia (MAguire et al., 2011; Maguire et al., 2017). Additionally, in 2012 the signal crayfish was recorded in a continental karstic river, the Korana River, where it was intentionally, and illegally, introduced (Hudina et al., 2013; Hudina et al., 2017; Maguire et al., 2018).

\section{Procambarus fallax (Hagen, 1870) f. virginalis - marbled crayfish (Croatian: mramorni rak); Figs. 1h, 6}

The taxon Procambarus fallax $\mathrm{f}$. virginalis (the name listed on the Union list) was recently described as a separate species by Lүко (2017), and its valid taxonomic name is Procambarus virginalis Lyko 2017. It was originally discovered in the German aquarium pet trade (MARTin et al., 2010a; MARtin et al., 2010b; MARTin et al., 2016; Lyко 2017) and considered a parthenogenetic form of the slough crayfish, P. fallax (MARTin et al., 2010a; IUCN, 2018). Its morphological characteristics are marbled color pattern and small chelae. It is usually less than $10 \mathrm{~cm}$ long, although some individuals can grow up to 12 cm total length (Vogt et al., 2004; CABI, 2018g). It is closely related to P. fallax, which is known from peninsular Florida and southern Georgia in USA, where it occurs in 
variety of lentic and lotic habitats such as forested and open wetlands, ponds, and ditches (MARTin et al., 2010a; IUCN, 2018). However, established populations in Europe are found in lentic habitats only, such as gravel pit lakes near urban areas (CHUCHOLL et al., 2012).

The main pathway of its introduction is the deliberate release of aquarium specimens (Chucholl, 2014 and references therein). This was also the presumed pathway of its introduction to Croatia (SAMARDžić et al., 2014). Since its discovery in the wild in Europe from the beginning of 2000, P. fallax $\mathrm{f}$. virginalis has established populations in Germany, the Netherlands, Italy, Slovakia, Croatia, Sweden, Ukraine, and Hungary (Marzano et al., 2009; Chucholl et al., 2012; Novitsky \& Son, 2016, Lóккös et al., 2016).

The parthenogenetic breeding mode is not known in any other decapod crustacean (Martin et al., 2010a). The consequent high fecundity and extended breeding period, as well as a fast growth rate, make marbled crayfish populations difficult to control even in aquarium (Novitsky \& Son, 2016). Those traits, along with a wide range of ecological tolerances, give them competitive advantages over the native crayfish species, whom they displace from their habitats (CHucholl et al., 2012 and references therein). They are known vectors of the crayfish plague pathogen Aphanomyces astaci, which can also negatively impact native crayfish species (Keller et al., 2014).

The first and presently only known record of marbled crayfish in Croatia is the Šoderica gravel pit lake, where an established population was found in 2014 (SAMARDžIć et al., 2014; MAguire et al., 2018). Considering its high invasiveness and the documented negative impacts of other alien crayfish species on native crayfish, it is to be expected that the marbled crayfish will accelerate the decline of native crayfish in Croatia.

Eriocheir sinensis H. Milne-Edwards, 1853 - Chinese mitten crab (Croatian: kineska rakovica)

The species Eriocheir sinensis is currently not present in Croatia. However, it was found in the Serbian section of the Danube River (ŠKRABA et al., 2013), so its spread to Croatian part of the Danube is likely and it is considered a door knocker species.

\section{Freshwater fish}

Two freshwater fish species of Union concern are present in Croatia: Pseudorasbora parva Dybowski, 1877 and Perccottus glenii (Temminck \& Schlegel, 1846). While P. par$v a$ is widely distributed through freshwater systems in Croatia, $P$. glenii was found only once.

Perccottus glenii Dybowski, 1877 - Amur sleeper, Chinese sleeper (Croatian: rotan); Figs. 2a, 7

The species Perccottus glenii is a small to medium sized freshwater fish species with body size up to $250 \mathrm{~mm}$ and weight up to $250 \mathrm{~g}$. Body is covered with scales, without lateral line canals, green to gray in color, except in spawning period when males become almost completely black with green spots (KотTELAT \& FreyHOF, 2007). It inhabits stagnant freshwater habitats such as lakes, ponds, reservoirs, gravel pits, irrigation channels and floodplains. Flowing waters (rivers, canals) are not a common habitat for P. glenii, but they are an important pathway for its unaided spread (long-distance corridors). $P$. glenii is not a very good swimmer, which is why they usually spread more rapidly downstream then upstream (Reshetnikov, 2013). 
The native range of $P$. glenii is northeast Asia (Reshetnikov \& Ficetola, 2011; ReshetNikov, 2013). The first record from Europe dates back to 1912 when it was transported to St. Petersburg province in Russia for aquarium purposes since which time it has been introduced widely across Europe and Asia. In Europe it was recorded in Russia, Belarus, Ukraine, Lithuania, Latvia, Estonia, Finland, Poland, Germany, Slovakia, Hungary, Serbia, Croatia, Bosnia and Herzegovina, Bulgaria, Romania, and Moldova (Reshetnikov \& Ficetola, 2011; Reshetnikov, 2013; CABI, 2018h). The main pathway of introduction is intentional transport of live fish for aquaria. They were usually kept in garden ponds from which they could easily escape to open waters. Other pathways include intentional or unintentional translocation by local people and unaided spread through water corridors. Also, unintentional introductions through contamination of fish farming stocks (usually of Cyprinus carpio) are recorded (RESHETNIKOv \& FiceTOLA, 2011; Reshetnikov \& Schliewen, 2013; Nehring \& Steinhof, 2015; CABI, 2018h). The spatio-temporal dynamics of $P$. glenii records in Eurasia confirms colonization from more than one center of invasion (RESHETNIKov, 2013).

Many authors declare $P$. glenii a 'perfect conqueror' because of its reduced size, wide thermal tolerance, opportunistic diet (from ciliates to vertebrates, including fish eggs and small fish), and aggressive behavior (Koščo et al., 2008; ĆALETA et al., 2011; LuCA \& GHIorghíŢĂ, 2014). Because of its high invasiveness, the lack of geographical barriers and the existence of appropriate climate conditions in Europe, as well as absence of reliable prevention methods the species has a very high risk of expansion through the Europe (Reshetnikov, 2013). It negatively affects native species through competition and predation. Decrease in relative abundance of native species, such as Carassius carassius, Leucaspius delineatus, Rhodeus amarus, and Umbra krameri, are reported for some parts of the non-native range of $P$. glenii (Reshetnikov, 2013; CABI, 2018h).

In Croatia, only one specimen of $P$. glenii has been recorded so far. This specimen was caught by an angler in the River Sava near Slavonski Brod in July 2008, in a canal connected to the carp fishpond (ĆALETA et al., 2011). Since then, despite the significant capture effort, no P. glenii has been caught in Croatia. Three hypotheses concerning the introduction of the species to the Croatian waters are proposed: (i) transfer of the fish from Hungary or Serbia to the fish ponds followed by their escape to the open waters; (ii) natural dispersal from the Danube during spring flooding and (iii) via ballast water of ships (ĆAleta et al., 2011). According to Piria et al. (2016), this species has high invasiveness potential in Croatia.

\section{Pseudorasbora parva (Temminck \& Schlegel, 1846) - topmouth gudgeon, stone morroco (Croatian: bezribica, amurski čebačok); Figs. 2b, 7}

The species Pseudorasbora parva is the only representative of the fish genus Pseudorasbora known in Europe. The body is up to $110 \mathrm{~mm}$ long, greenish gray or silver in color. During spawning period males develop bluish or purple-gray coloration and nuptial tubercles on snout (KotTelat \& FreYhof, 2007). Mouth is small and superior, without barbels. The species inhabits a wide variety of habitats, but it is most abundant in shallow, densely vegetated ponds, small lakes, and canals, where the female lays eggs on vegetation. Females have high fecundity and spawn multiple times in season, while males guard nests until fry is hatched (Kottelat \& FreyHof, 2007). It is an opportunistic omnivore, feeding on algae, plant material, zooplankton, insects, crustaceans, fish eggs, and small fish (YALÇıN-ÖZdiLEK et al., 2013). 


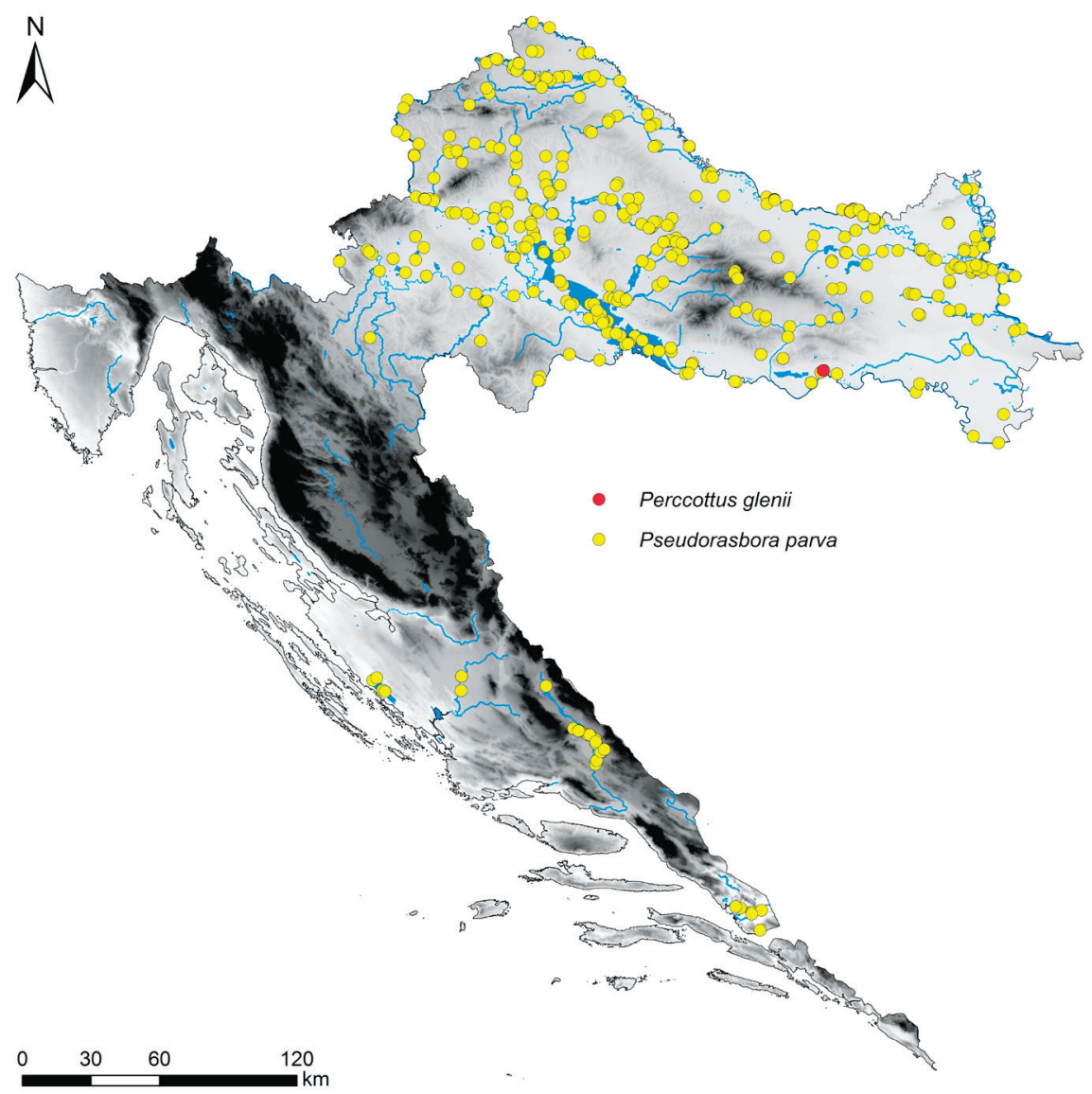

Fig. 7. Distribution of Perccottus glenii (Temminck \& Schlegel, 1846) and Pseudorasbora parva Dybowski, 1877 in Croatia

Its native range is northeastern Asia, from the river Amur to the Pearl River drainages in Siberia, Korea, China, and Japan but has been widely introduced to various areas in Asia and Europe (Kotтelat \& Freyhof, 2007; CABI, 2018i). The introduction to the European waters was accidental - the species was unintentionally shipped from China along with the herbivorous Chinese carp fry to the fish farms in Europe (Romania, Lithuania, Hungary and Ukraine) in the early 1960s (Welcomme, 1988; Golzan et al., 2010; W IткошsкI, 2011). Since its initial introduction, it invaded almost all countries in Europe as stock contaminant, through intentional introduction as ornamental fish or fish bait, and through secondary spread to new areas. It is especially abundant in Eastern Europe, from Danube to Kuban drainages, east to Volga and south to central Iran (KotTElat \& FREYHOF, 2007).

Strong negative effects of $P$. parva on habitats and species have been recorded (WITKowsKI, 2011). When in high densities, it competes with other species for food. Through predation on larger zooplankton it increases phytoplankton densities and, subsequent- 
ly, increases eutrophication of water. Also, it can actively predate upon fish eggs and fry of native species, reducing their abundance. It is also a vector of several parasites representing a threat to native and farmed fish species (WITKOWsKI, 2011).

The species P. parva was noticed in mid 1980s in Croatia (TVRTKović, 1985; HABEKović \& Popović, 1991) where it was probably introduced through fish farming stocks or by unaided spread from rivers in the Danubian basin. The species is currently distributed throughout the entire country in waters of both the Adriatic and the Danubian drainages. In Croatia, it occurs in high abundance in adequate habitats, especially in waters belonging to Danubian drainage and near fish farms. According to PIRIA et al. (2016), it has medium high invasiveness potential in Croatia.

\section{Reptiles}

Only one invasive alien reptile species of Union concern is present in Croatia Trachemys scripta (Schoepff, 1792).

Trachemys scripta (Schoepff, 1792) - pond slider, common slider (Croatian: crvenouha kornjača (T. s. elegans), žutouha kornjača (T. s. scripta)); Figs. 2c, 8

The species Trachemys scripta is medium sized terrapin with carapace length up to 280 mm (BRINGsøe, 2006). Coloration of carapace and skin is olive to brown with yellow stripes and spots. It has yellow or red patches on each side of the head (BRINGSøE, 2006; CABI, 2018j). The species is a freshwater habitat generalist but prefers larger, stagnant, well vegetated water bodies with soft bottoms and suitable basking sites (BRINGSøE, 2006).

The native range of the pond slider includes the southeastern United States and northeastern Mexico, where species is represented with three subspecies - T. s. scripta, T. s. elegans, and T. s. troostii. Because they are among the most popular pet animals, non-native distribution of pond sliders is almost the whole of the world, including almost all European coutries (BRingsøe, 2006; Jelić \& Jelić, 2015; CABI, 2018j).

The main pathway of introduction of T. scripta worldwide is pet release into seminatural and natural habitats. The most popular subspecies in pet trade is T. s. elegans, which was in the aquarium trade market in massive numbers from the 1970s. After bans on selling this particular subspecies in Europe in 1997 (EC, 1997), it was replaced either with other subspecies (usually T. s. scripta) or other similar species of terrapin (BRINGSøE, 2006; CABI, 2018j). Records of T. scripta in natural habitats in Europe date back to the 1980s (BRINGSøE, 2006), while its first official record in Croatia is from 1999 from a pond near Grubišno polje (Jelić \& Jelić, 2015; Jelić et al., 2016). Since then many individuals were recorded in Croatia, usually in ponds and lakes in public parks (KoREN et al., 2018). The largest known population of T. scripta in Croatia, with around 300 individuals, is in Maksimir park's ponds in Zagreb (Jelić \& Jelić, 2015; Jelić et al., 2016). Despite some indications that it is unable to reproduce in the wild in Europe, records of egg-laying females and young individuals (probably hatchlings) were recorded on several occasions in Croatia and countries with similar climate (Serbia, Spain) indicating that climate conditions in Croatia are suitable for its successful reproduction (PEREzSantigosa et al., 2008; ĐorĐević \& AnĐelković, 2015; Schweiger, 2015; Jelić et al., 2016). Taking this into account, it is likely that T. scripta has established in Croatia. Because of its negative impact on native species through competition (with native Emys orbicularis and Mauremys rivulata), predation and potential disease transmission, it is considered invasive in Croatia. It can impact human health through possible transmission of human salmonellosis (NAGANo et al., 2006; LAFUENTE et al., 2013). 


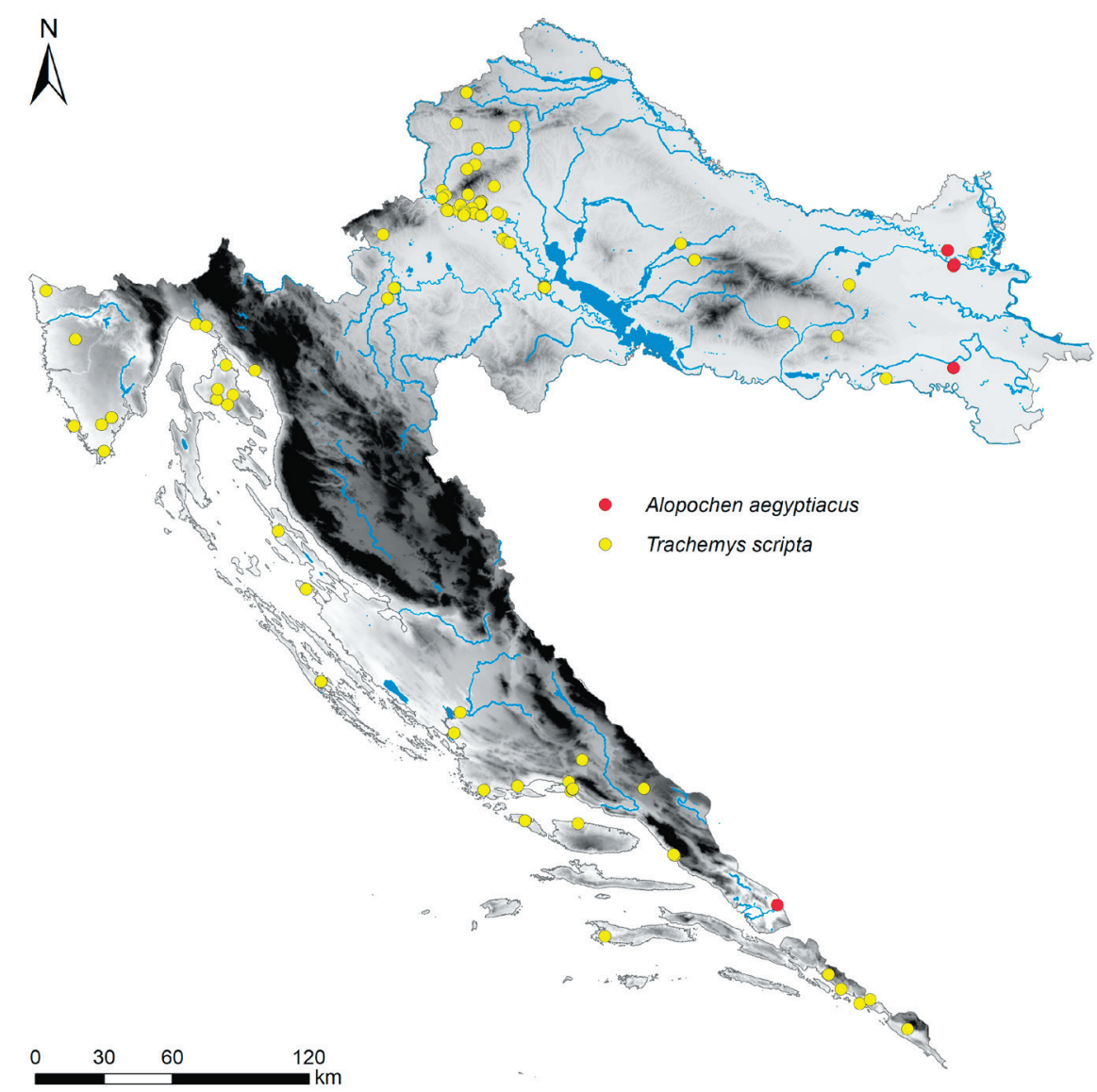

Fig. 8. Distribution of Trachemys scripta (Schoepff, 1792) and Alopochen aegyptiacus (Linnaeus, 1766) in Croatia.

\section{Amphibians}

Lithobates catesbeianus (Shaw, 1802) - American bullfrog (Croatian: sjevernoamerička žaba bukača)

The status of the amphibian species Lithobates catesbeianus in Croatia is currently uncertain. There is one literature record of the American bullfrog in Croatia (JANEv-HuTINEC et al., 2006), but published data about time or place of this record are lacking. Most likely, the species was introduced as a stock contaminant in one fish farm near Karlovac before 2006 (Burić et al. 2018, N. TVRTKоvić, pers. comm.), but further confirmation is necessary. Recently it was found in Slovenia near the border with Croatia (Kirbiš et al., 2016). Because of climate and habitat suitability and the proximity of the recent finding in Slovenia it can be considered a door knocker species for Croatia.

\section{Birds}

In Croatia only one invasive alien bird species of Union concern is present - Alopochen aegyptiacus (Linnaeus, 1766). 


\section{Alopochen aegyptiacus (Linnaeus, 1766) - Egyptian goose (Croatian: egipatska guska); Figs. 2d, 8}

The species Alopochen aegyptiacus is the only representative of the bird genus Alopochen (family Anatidae). It has pink legs and bill, dark brown upper wings and head, a dark patch around eyes and in the middle of chest, while the rest of the plumage is light brown (Heinzel et al., 1995; Gyimesi \& Lensink, 2010). It is mainly a bird of large rivers and floodplains, but it can inhabit a broad range of wetland habitats, such as ponds, lakes, swamps, estuaries, and wet meadows (Sсотт \& Rose, 1996; Callaghan, 2016). Its diet includes grass, aquatic vegetation, seeds, and a wide range of agricultural crops (Halse, 1984; Callaghan, 2016). It can nest on the ground, under the vegetation, in trees, cliffs, and in caves, as well as in buildings and houses (Pitman, 1965).

It is native to sub-Saharan Africa, where it is one of the most common and widespread waterfowl (SMith, 2012; CALlaGHAN, 2016). It was introduced to Great Britain at the end of $17^{\text {th }}$ century as an ornamental waterbird, and it had become established in the wild by the $18^{\text {th }}$ century (Lever, 2005). On the continent, a breeding population was first observed in the Netherlands near The Hague in 1967 (Lensink, 1999). The Egyptian goose is a highly territorial and adaptable species. It is currently established and widely distributed in Great Britain, Belgium, the Netherlands, Germany, and France (BANKs et al., 2008), and is considered one of the most rapidly spreading invasive species in Europe (Lensink, 1999; Smith, 2012; Callaghan, 2016).

It is considered a serious agricultural pest throughout its native range, especially of cereal crops (MANGAL \& Crowe, 2002; Gyimesi \& Lensink, 2010). The species can negatively impact native bird species through competition for food and nesting sites (GYIMESI \& LENSINK, 2010) and through hybridization with other geese (e. g. Branta canadensis and Anser anser) or ducks (e. g. Anas platyrhynchos; BAnks et al., 2008), although the hybrids are usually sterile (Homma \& GeITER, 2010). Furthermore, if population density is high, the large amounts of the bird's faeces can cause the eutrophication of freshwater habitats, and the birds can be a threat to aircraft safety if they live and fly near airports (LENSINK et al., 2010). They are also a known vector of avian influenza (Shihmanter et al., 1998).

In Croatia it was recorded near Slavonski Brod (KRALJ, 2013), Darda, Metković and Osijek (BARIšıć et al., 2016). The data about established populations in Croatia do not exist.

\section{Mammals}

Five invasive alien mammal species of Union concern are currently known for Croatia: Herpestes javanicus (É. Geoffroy Saint-Hilaire, 1818), Myocastor coypus (Molina, 1782), Nyctereutes procyonoides (Gray, 1834), Ondatra zibethicus (Linnaeus, 1766), and Procyon lotor (Linnaeus, 1758).

Herpestes javanicus (É. Geoffroy Saint-Hilaire, 1818)* - small Indian mongoose, small Asian mongoose, Javan mongoose, Indian mongoose (Croatian: mali indijski mungos); Figs. 2e, 9

\footnotetext{
* The name Herpestes javanicus on the Union list refers to the subspecies introduced to Croatia, H. j.auropunctatus, which is by some authors considered a separate species $H$. auropunctatus, see Taxonomic notes below
} 
The small Indian mongoose (H. j. auropunctatus; see Taxonomic notes below) is the smallest of all Asian Herpestes species. It has a slender body with short legs and robustly muscular tail. The head is elongated with a pointed muzzle and short ears. Feet are pentadactyl with long sharp non-retractile claws. Fur is short and soft, pale to dark brown, and flecked with golden spots (Nellis, 1989). Males have a longer and more robust body and wider head than females (Nellis, 1989). Adult body mass ranges from 300 to $900 \mathrm{~g}$ (Hays \& ConAnt, 2007). Mongooses are generalist feeders whose diet changes seasonally and locally (Simberloff et al., 2000). They are diurnal and terrestrial animals - they can swim and climb trees but they will avoid water whenever possible, they reduce their activity during rainy periods and will not voluntarily enter water deeper than $5 \mathrm{~cm}$ (Nellis \& Everard, 1983). They can live in various habitats such as forests, grasslands, wetlands, deserts, and coastal and riparian zones, as well as in agricultural areas, landfills, ruderal and urban areas (NeLLIs \& EverARD, 1983; GISD, 2018).

The small Indian mongoose is native to the Middle East and much of South and Southeast Asia (NeLLIS, 1989). It was intentionally introduced more widely than any other mammal (HAYs \& ConANT, 2007). It was successfully introduced to at least 64 islands in the Pacific and Indian Oceans and the Caribbean and Adriatic Sea, and to the continental South America and Europe, however introductions to North America and Australia were unsuccessful (BARUN et al., 2011a). All documented introductions of this species were intentional, because of its reputation as a control agent for rats and snakes. Unfortunately, it is also an efficient predator of many endangered and threatened vertebrates (Hays \& Conant, 2007).

The small Indian mongoose was first introduced to Croatia in 1910 to the island of Mljet for control of the poisonous horned viper (Vipera ammodytes) populations, when seven males and four females from India were released. As a consequence, the native horned viper has been extinct on Mljet since World War II (Tvrtкović \& KRYšTufeK, 1990). It was subsequently successfully introduced to several nearby islands: Korčula (in 1921), Hvar (ca 1970s), Čiovo (ca 1950s), Škrda near Pag Island (ca 1950s), Hvar (ca 1970s), and Kobrava near Mljet Island (Tvrtković \& KryštufeK, 1990; KrYštufeK \& TvrtKović, 1992; BARUn et al., 2008; BARUn, 2011), as well as on the islets of Moračnik, Ovrata and Tajnik near Mljet Island (SELANEC et al., 2016). It was also unsuccesfully introduced to the island of Brač (in 1926) (TvrTKović \& KRYšTufeK, 1990). It was introduced to Pelješac Peninsula repeatedly from 1921 to 1927 (TvrtKović \& KrYšTufeK, 1990). Since then it spread along the southern Dalmatian and Montenegro coast up to Neretva River in the Bosnia and Herzegovina in the north and Albania in the south (BARUN et al., 2011b; Ćirović et al., 2011, Ćirović \& ToholJ, 2015). At present, the European range of the species includes the Adriatic coast (Fig. 9) from the island of Škrda and the Neretva River in the north, to Albania in the south (GAUBERT, 2016).

The small Indian mongoose is a generalist predator causing the decline and extirpation of native mammals, birds, reptiles, and amphibians on islands (Lowe et al., 2000; BARUn, 2011). On Croatian islands it is harmful to the wild fowl and it damages vegetables, figs, grapes as well as poultry (Tvrtкović \& KryštufeK, 1990). Additionally, it carries human and animal diseases, including rabies and human Leptospira bacterium (Pimentel, 1955; Nellis \& Everard, 1983). Contrary to what might be expected, the residents on the island of Mljet like the mongoose and they do not see it as a pest. Furthermore, it is considered a tourist attraction (GJuRAšıć et al., 2014). 


\section{Taxonomic notes}

The taxonomy of H. javanicus is unclear and often debated. From 1940s some authors considered Herpestes javanicus (É. Geoffroy Saint-Hilaire, 1818) and Herpestes auropunctatus (Hodgson, 1836) separate species, while others considered it a single species, $H$. javanicus, with $H$. j. auropunctatus as one of the subspecies (for more details see VeroN et al., 2007). In recent molecular studies (Veron et al., 2007; Patou et al., 2009; Veron \& JENNINGs, 2017) phylogenetic analyses showed that $H$. javanicus, $H$. auropunctatus, and their closest relative, the sympatric H. edwardsii (É. Geoffroy Saint-Hilaire, 1818), are molecularly distinct clades (although with unclear relationships among them). Separation of $H$. auropunctatus from $H$. javanicus is supported by morphological evidence according to some authors (TAYLOR \& MATHESON, 1999), while others consider this an intraspecific size variation due to competition or the lack of it (SIMBERLOFF, 2000).

The name used on the Union list refers to the subspecies H. javanicus auropunctatus, commonly called the small Indian mongoose. The small Indian mongoose is found from the Arabian Peninsula across the northern Indian subcontinent to Southeast Asia (Gilchrist et al., 2009), and this is the taxon (either species or subspecies) that was introduced at the end of the $19^{\text {th }}$ and during the $20^{\text {th }}$ century to many different parts of

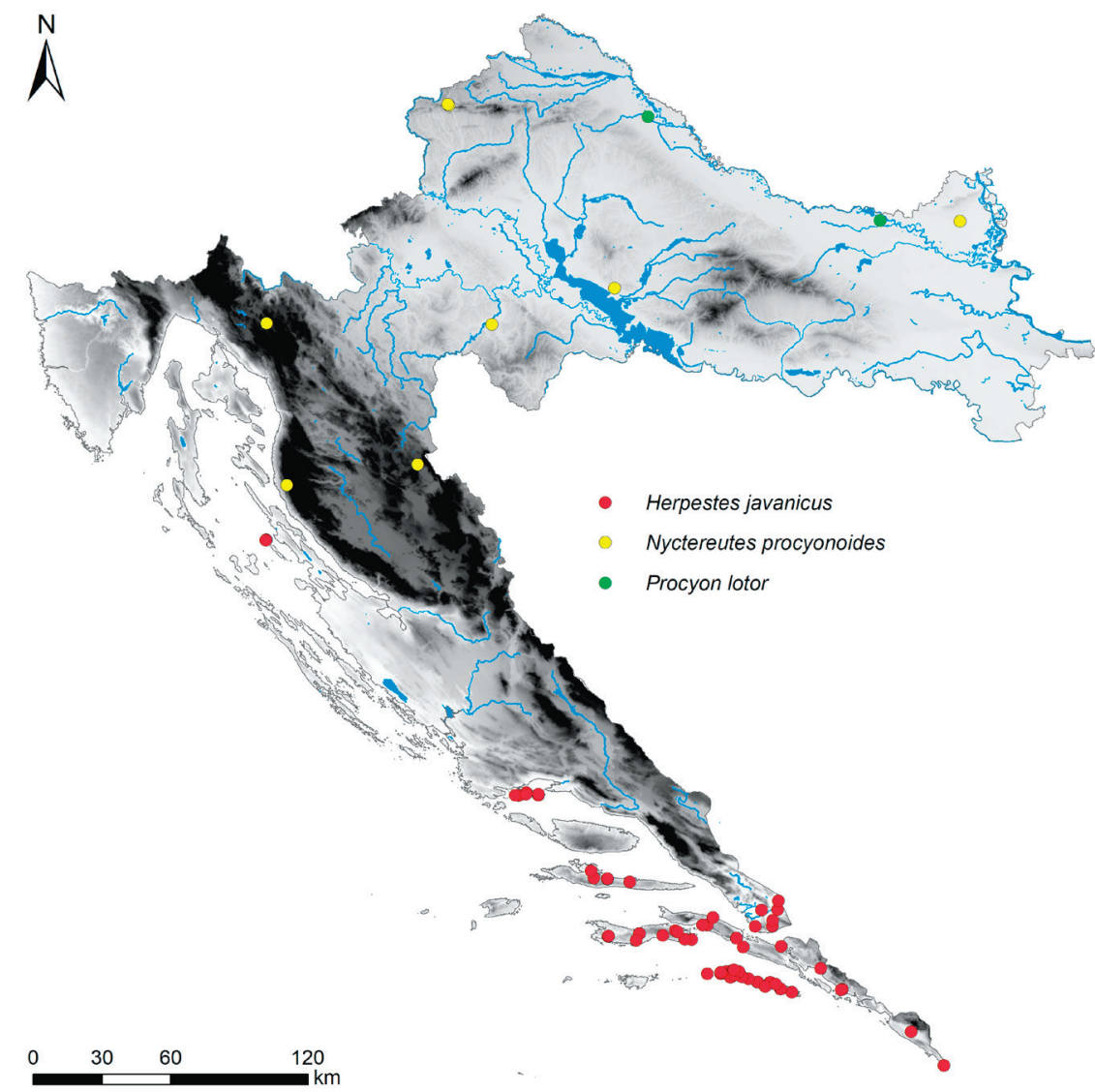

Fig. 9. Distribution of Herpestes javanicus (É. Geoffroy Saint-Hilaire, 1818), Nyctereutes procyonoides (Gray, 1834), and Procyon lotor (Linnaeus, 1758) in Croatia. 
the world (mainly on islands) for the biological control of rats and snakes in plantations (Tvrtkovic \& KrYštufeK, 1990; Simberloff et al., 2000; Barun, 2011; Veron \& Jennings, 2017). The remaining populations (or separate species H. javanicus) are geographically separate from the small Indian mongoose, occurring from Java through Sumatra and mainland South-east Asia.

\section{Myocastor coypus (Molina, 1782) - coypu (Croatian: nutrija); Figs. 2f, 10}

The species Myocastor coypus is a rat-like semiaquatic rodent, the only member of the genus Myocastor (Woods et al., 1992). It has a robust body, covered with light to dark brown dense fur, and bright orange incisor teeth. The average body length, not including the tail, is 52 centimeters. Its tail is long (average $37 \mathrm{~cm}$ ), rounded, and covered with sparse hairs, which distinguishes it from beavers and muskrats. It was traditionally classified as the only member of the family Myocastoridae, with unclear relatedness to other rodent taxa (Woods et al., 1992), but recent molecular phylogenetic analyses revealed it belongs to the family Echimyidae, South American spiny rats (FABRE et al., 2013; Loss et al., 2014). Coypu inhabits waterways, marshes, rivers, lakes, and prefers sites with an abundance of emergent aquatic plants, their main food source. They can also occasionally feed on invertebrates, such as freshwater mussels (BARосн et al., 2002; KumE et al., 2012), and on eggs of ground nesting birds (WINDHOFFER, 2017). They are nocturnal, but they can become diurnal during the cold periods (Woods et al., 1992). They construct 1-6 meter long burrows in banks near the water, sometimes with several entrances.

The species is native to the southern half of South America and is introduced worldwide (Woods et al., 1992). Extensive fur farming of coypu was established in Europe and North America in the first half of the $20^{\text {th }}$ century, and since then it was introduced to many countries in Europe, Asia, Africa, and North America, often establishing in the wild (Woods et al., 1992; CARTER \& LEONARD, 2002). The main pathway of introduction is intentional or accidental release from fur farms. Occasionally they were also released for the biological control of water weeds (CARTER \& LEONARD, 2002).

In the non-native habitats it can reach very high population densities. Main impacts are destruction of marsh habitats by feeding on native vegetation and disruption of irrigation systems due to the burrowing activities in the river banks and ditches (Woods et al., 1992; CARTer et al., 1999; CARTER \& LeONARD, 2002). It can cause damage to agriculture by feeding on crops (Linscombe \& KinLer, 1997; Guichón et al., 2003) and will decrease water bird populations by nest disturbance (BERTOLINo et al., 2011; ANGELICI et al., 2012). Also, it can transmit parasites and disease to livestock, wildlife, and humans (Gebhardt, 1996; Moutou, 1997; Michel et al., 2001; Fratini et al., 2015).

In Croatia the coypu was first found in 1936, in marshland near Donja Dubrava, Međimurje (Hirtz, 1937a). The species was introduced to Croatia by spread from neighbouring countries (Slovenia, Hungary or Austria), where it was released or escaped from the fur farms. An established population in the Mirna river valley in Istria was known prior to 1989 (PURGER \& KRYŠTUFEK, 1991), and recent records also include the river basins of the Sava, Drava, and Kupa rivers (Fig. 9). According to the Animal Protection Act (Anonymous, 2017) fur farming is prohibited in Croatia, so currently the only pathway for introduction to new habitats is the unaided spread of the population. Its eradication has been allowed in the Mirna valley since 2008, but the population established there has not been completely eradicated. In other countries complete eradication was shown to be less costly than long-term population control (GosLing et al., 1988; PANZACCHi et al., 2007). Known impacts of M. coypus in Croatia are destruction of native vegetation and damage of river banks and irrigation systems. 


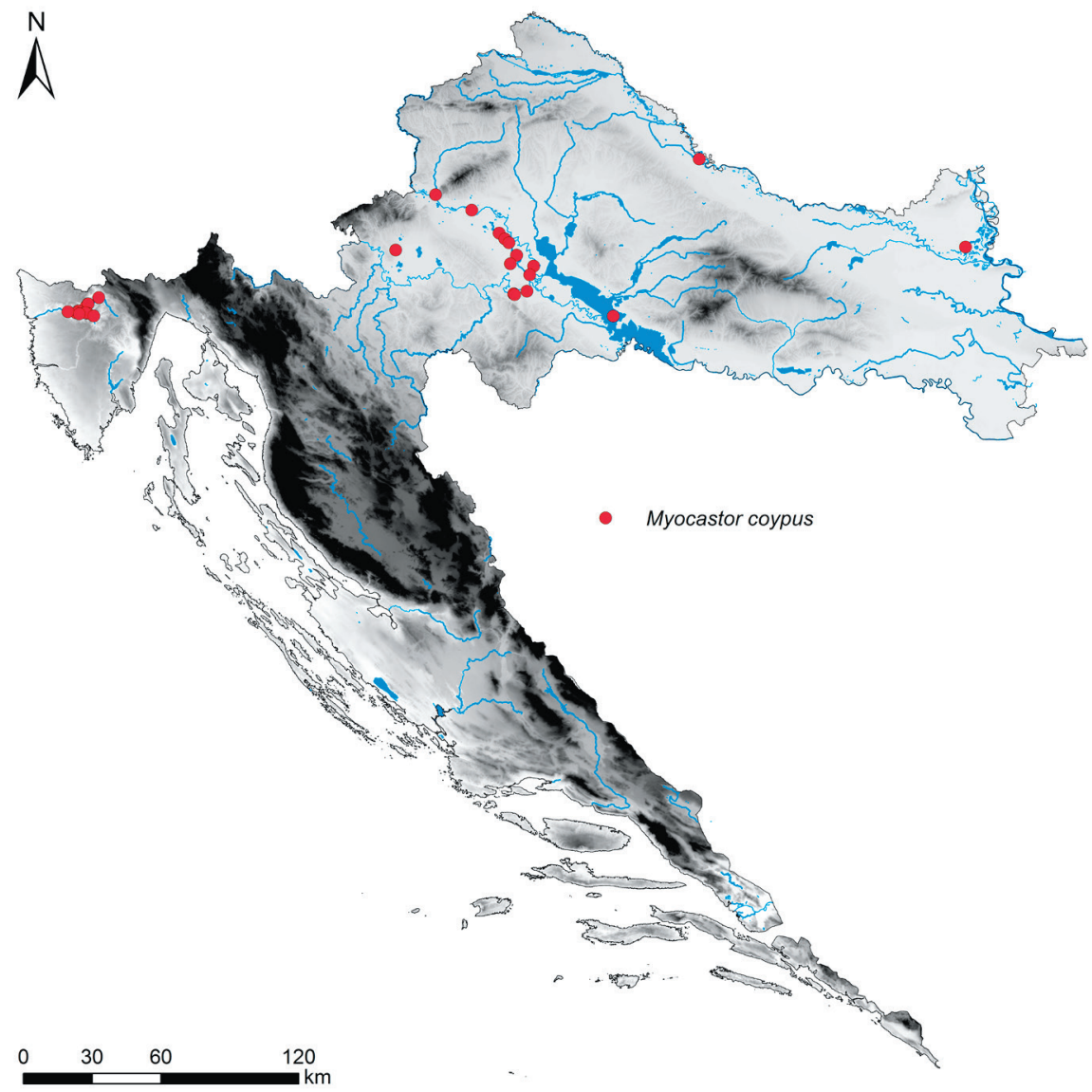

Fig. 10. Distribution of Myocastor coypus (Molina, 1782) in Croatia.

Nyctereutes procyonoides (Gray, 1834) - raccoon dog (Croatian: rakunopas, kunopas); Figs. 2g, 9

The species Nyctereutes procyonoides is a member of the Canidae family, about the size of a small fox (body length $57-71 \mathrm{~cm}$ ) but with shorter legs, with thick, long and dense fur, light grey to black in color, and with a distinct black mask around the eyes (WARD \& WURSTER-HiLL, 1990). It inhabits variety of habitats, from meadows and forests with dense undergrowth to mosaic of agricultural land, and is often found near the water (KauHaLa, 1996).

Originally from Eastern Asia it was brought to Europe for fur production. An exceptionally large number of individuals (9100 animals) were introduced between 1929 and 1955 to multiple locations in the European part of the former Soviet Union and were often deliberately released from fur farms (KauHala \& KowALCZYK, 2011). They became established in the wild and dispersed rapidly across northern and eastern Europe (KowalczyK, 2006; Kauhala \& KowalczyK, 2011). Today they are established in Belarus, Bulgaria, Estonia, Finland, Germany, Latvia, Lithuania, and Poland (CABI, 2018k). The success of their establishment and spread in non-native range is attributa- 
ble to the high genetic variability of introduced population, high reproductive capacity, its ability to hibernate during the winter in the cold climates, an opportunistic diet, and high dispersal ability (Sutor et al., 2010; Kauhala \& KowalczyK, 2011).

The severity of its impact on the native ecosystems is somewhat controversial because of a small number of studies experimentally demonstrating clear effects (CABI, 2018k). When their population is dense it negatively impacts, through direct predation, native birds, amphibians, reptiles, and small mammals; while native carnivores are affected through competition (KowAlCZYK, 2006; Sutor et al., 2010; Kauhala \& KowaLCZYK, 2012). Furthermore, it impacts wild and domesticated animals through the transmission of rabies (KowALCzYK, 2006; Singer et al., 2009) and parasites (OKSANEN et al., 1998; Oivanen et al., 2002).

In Croatia, most likely pathway of introduction was migration from neighboring countries, from Serbia, Hungary, and Slovenia (Ćirović \& Milenković, 1999; Kauhala \& KowAlCzYK, 2011; Budinski et al., 2018). The individual records (Fig. 9) are scattered across Croatia and they include Baranja (PAvlović, 1994, cited by Ćirović \& Milenković, 1999), the Dinarides near Mrkopalj (Duplić et al., 2016), Plitvice Lakes National Park (N. MAGdić, pers. comm.), and Northern Velebit area (Śver et al., 2018). This is probably a reflection of the species' high dispersal capacity, but the presence of established populations is not confirmed. No data about the impact of the raccoon dog on native species and habitats exist for Croatia.

\section{Ondatra zibethicus (Linnaeus, 1766) - muskrat (Croatian: bizamski štakor); Figs. 2h, 11}

The species Ondatra zibethicus is a semiaquatic rodent, similar to Myocastor coypus but smaller, with 45-55 cm average body length, including a long, hairless, laterally flattened tail (Willner et al., 1980). The species is the only representative of the genus Ondatra (family Cricetidae) (STEPPAN \& SCHENK, 2017). It inhabits marshes, lakes, ponds, and other slow moving waters, including brackish and saline systems, but prefers eutrophic waters with plenty of vegetation (Willner et al., 1980; SMirnov \& TRETYAKov, 1998). For their nesting site muskrats excavate burrows in the banks and construct lodges in the water out of plant material, with one or several chambers above the water level. In most cases, they use burrows in summer and lodges during winter (CABI, 20181).

The native range of $O$. zibethicus covers most of North America. They were brought over to Europe in 1905 for fur farming, where they quickly established in many countries, thanks to their high reproduction rate, good dispersal ability, and wide climatic tolerance (Skyriene \& Paulauskas, 2012; CABI, 20181). Muskrat fur farms do not exist anymore in Europe, so the only current introduction pathway to the new areas is secondary unaided dispersal. Within Europe it has established populations in Austria, Belgium, the Czech Republic, Finland, France, Germany, Hungary, the Netherlands, Poland, Russia, Sweden, Switzerland, and Bosnia and Hercegovina (SKrrienè \& PAuLAUSKAS, 2012; CABI, 20181). It was successfully eradicated from the United Kingdom and Ireland (DANELL, 1996).

Large populations of $O$. zibethicus can damage river banks and flood dikes with their burrowing activities, and diminish aquatic plant communities by grazing, which also causes the loss of nursery sites for fishes (SKYrienE \& PAUlausKas, 2012). Muskrats predate on freshwater bivalves and crustaceans which can cause their population to decline, occasionally damage agricultural crops, and can have negative impact on na- 
tive semi-aquatic rodent populations because of competition for resources (GEBHARDT, 1996; Owen et al., 2011; Skyrienė \& Paulauskas, 2012; Birnbaum, 2013).

The muskrat was first found in Croatia in 1932 (DolEnEC, 1934), introduced from Hungary and Austria by dispersal down the Mura River (Plančić, 1937), and since then it has spread across the country (Fig. 11), in the majority of the large rivers: Danube, Sava, Kupa, Drava, and Krka (Kaman, 1950; Savić, 1962; De Luca et al., 1989). Despite its long presence in Croatia there are no recent studies of its population density and size, or its ecological impact.

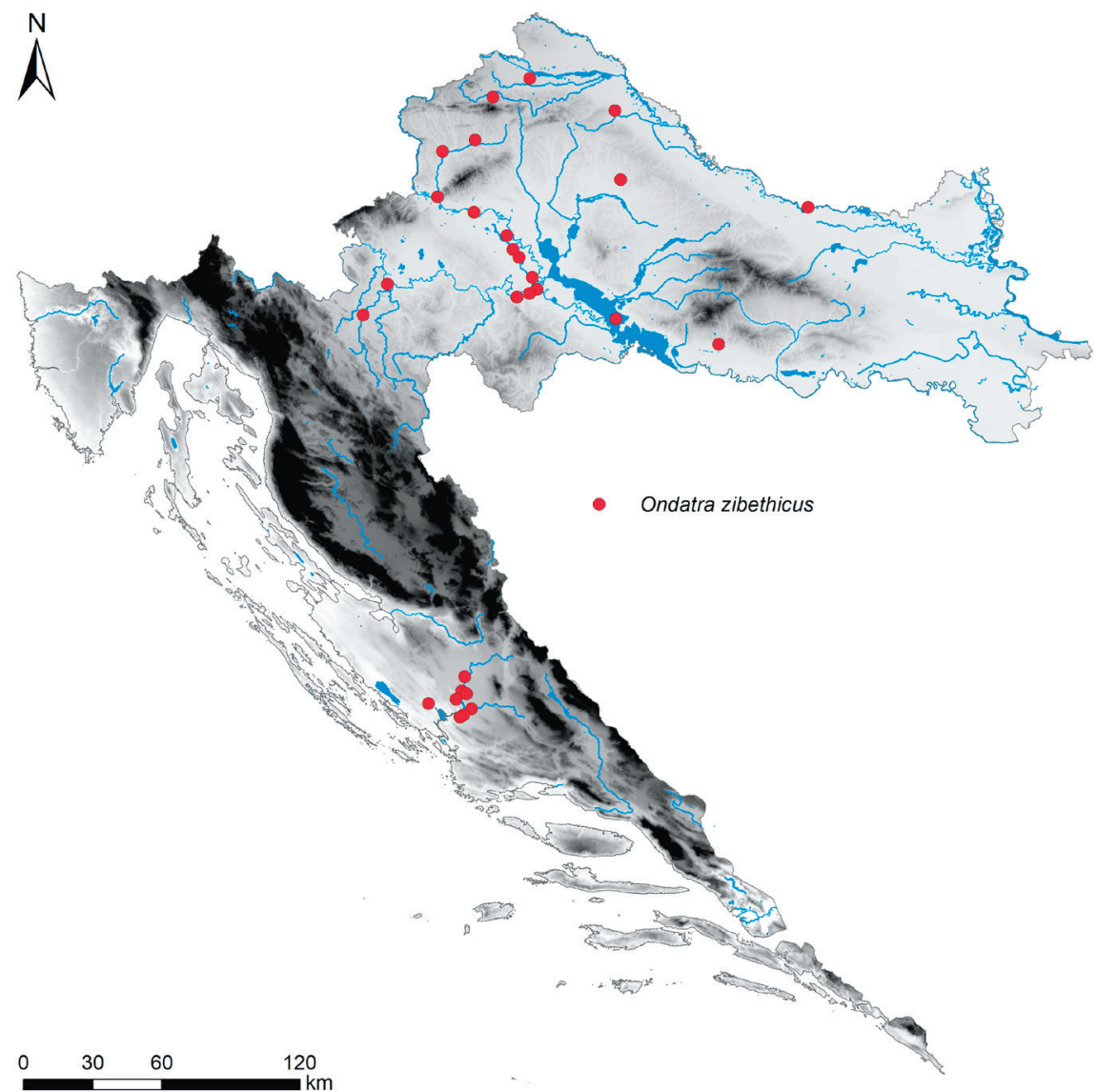

Fig. 11. Distribution of Ondatra zibethicus (Linnaeus, 1766) in Croatia.

\section{Procyon lotor (Linnaeus, 1758) - raccoon (Croatian: rakun); Figs. 2i, 9}

The species Procyon lotor is a medium sized carnivore, a member of family Procyonidae (LoTZE \& ANDERson, 1979), readily recognized by its brown to black mask around eyes, delimited with lighter, white-grey fur. The remainder of its coat is iron grey, varying to almost black or reddish, with lighter fur on legs, flanks, and tips of ears. Its tail is bushy and has five to seven characteristic white rings. Its feet have five digits each, with short claws, but no webbing. It is capable of using its front paws for grasping and manipulating objects (Lotze \& Anderson, 1979; BArtoszewicz, 2011). It eats a 
wide variety of plants and invertebrates (crabs, crayfish, molluscs), and predates on vertebrates, such as birds, turtles, mammals, and their eggs or young (BIEDRzYcKA, 2014). In urban habitats and in the absence of other food it will eat food remains from trash and cause damage in gardens and agricultural fields, which is why it is considered nuisance and pest in many cities (GEHRT, 2004).

Its native distribution ranges from southern Canada to Central America (GARCiA, 2012). In Europe, it was introduced to central Germany between 1927 and 1934. During the late 1960s, following the intentional release of an unknown number of individuals, it established in northern France (BARTOszewicz, 2006). It was also introduced to several locations in Russia and Belarus during the 1950s, but only a few populations succeeded in establishing there. The main pathways for raccoon introduction were intentional or accidental release from fur farms and intentional release for hunting, and more recently escapes of pet animals (ZALEWSKI, 2011). Because of its high plasticity in habitat selection and generalist diet it is now widely distributed throughout most of Central Europe, with large populations in some areas. For example, in Germany the population is estimated from 100,000 to one million raccoons (ZALEwski, 2011). Currently, in Europe the species is known for Austria, Belgium, the Czech Republic, Denmark, Great Britain, Hungary, Italy, Lithuania, Luxembourg, Liechtenstein, Montenegro, the Netherlands, Norway, Poland, Slovenia, Serbia, Slovakia, Sweden, and Switzerland (CAnova \& Rossi, 2008; CABI, 2018m).

In both the native and introduced range it causes major economic losses in urban areas by damaging fruit trees and garden crops, causing structural damage to houses, and damaging crops in the fields (IKEDA et al., 2004). It can negatively impact native bird fauna through egg predation, especially of waterfowls and larids (HARTMAN et al., 1997; Hartman \& EASTMAn, 1999; Ellis et al., 2007). Through both predation and competition it also impacts native mammals (IKEDA et al., 2004; ZALEWSKI, 2011), and it is a known vector of diseases and parasites for native fauna, domestic animals, and humans (GARcia, 2012).

In Croatia it was recorded recently at only two localities (Fig. 9): Podravski Podgajci (Bogdanovic, 2014) and Hlebine (K. Krapinec, pers. comm.). However, it was first recorded in 1937 when one individual was killed by a train in Stenjevec near Zagreb (Hirtz, 1937b). Considering its rare occurrence it is probably a casual species at this moment. No data about its impact on native species and habitats for Croatia exist.

\section{CONCLUSION}

Currently there are 49 invasive alien plant and animal species included on the Union list, out of which 17 species are present in Croatia. Three other plant species on the Union list might be present in cultivation, and two animal species are considered door knocker species in Croatia. While some of the present species are known only from one or few localities, others are widely distributed in Croatia. Depending on their presence and distribution, Regulation 1143/2014 prescibes prevention, early detection, rapid eradication or management. Therefore, for successful combat against invasive alien species of Union concern in Croatia, in order to prevent or mitigate their adverse impact, extensive work will be needed in the future, with the cooperation and concerted action of many other sectors (e. g. forestry, agriculture, fisheries, water management, customs). 


\section{ACKNOWLEDGEMENTS}

The data used in this paper were partly obtained through the project "Establishment of the National Monitoring System for Invasive Alien Species in Croatia" financed by EU Cohesion Fund in the framework of Operational Programme Competitiveness and Cohesion 2014 - 2020. We would like to thank our colleagues who allowed us to use their photos: Matija Franković for a photo of the small Indian mongoose, Sandra Hudina for a photo of Orconectes limosus, Luka Katušić for a photo of Pacifastacus leniusculus, Aleksandra Kočić for photo of Elodea nuttallii, Perica Mustafić for photos of Pseudorasbora parva and Perccottus glenii and Miroslav Samardžić for a photo of Procambarus fallax f. virginalis. We would also like to thank our colleague Stjepan Mekinić for providing his field data on Trachemys scripta in Split-Dalmatia County and the reviewers for improving our manuscript.

Received September 19, 2018

\section{REFERENCES}

Alekhnovich, A. \& Buňič, M., 2017: NOBANIS - Invasive Alien Species Fact Sheet - Orconectes limosus. - From: Online Database of the European Network on Invasive Alien Species - NOBANIS (www. nobanis.org). Date of access 22/01/2018

Anderson, L. W., 2005: California's reaction to Caulerpa taxifolia: a model for invasive species rapid response. Biological Invasions 7(6), 1003-1016.

Anonymous, 2017: Zakon o zaštiti životinja. Narodne novine 102/2017.

ANonymous, 2018: Zakon o sprječavanju unošenja i širenja stranih te invazivnih stranih vrsta i upravljanju njima. Narodne novine 15/2018.

BAgi, I., 2008: Common milkweed (Asclepias syriaca L.). In: Botta-Dukát, Z. \& Balogh, L. (eds.), The most important invasive plants in Hungary. Institute of Ecology and Botany, Hungarian Academy of Sciences, Vácrátót, Hungary. pp. 151-159.

Banks, A.N., Wright, L.J., Maclean, I.M.D., Hann, C. \& Rehfisch, M.M., 2008: Review of the Status of Introduced Non-Native Waterbird Species in the Area of the African-Eurasian Waterbird Agreement: 2007 Update. British Trust for Ornithology, The Nunnery, Thetford, Norfolk IP24 2PU.

BARIšIĆ, S., KRALJ, J. \& JuRInOvić, L., 2016: Rare birds in Croatia. The fourth report of the Croatian Birds Rarities Committee. Larus - Godišnjak Zavoda za ornitologiju Hrvatske akademije znanosti i umjetnosti 51(1), 46.

Baroch, J, Hafner, M., Brown, T. L., Mach, J. J. \& PochÉ, R. M., 2002: Nutria (Myocaster coypus) in Louisiana. Other Publications in Wildlife Management 46, 1-156.

Bartoszewicz, M., Okarma, H., Zalewski, A., Szczęsna, J., 2008: Ecology of the raccoon (Procyon lotor) from western Poland. Ann. Zool. Fennici 45, 291-29

Bartoszewicz, M., 2011: NOBANIS - Invasive Alien Species Fact Sheet -Procyon lotor- From: Online Database of the European Network on Invasive Alien Species - NOBANIS (www.nobanis.org). Date of access 10/01/2018.

BARUN, A., 2011: The small Indian mongoose (Herpestes auropunctatus) on Adriatic Islands: impact, evolution and control. PhD dissertation, University of Tennessee.

Barun, A., Budinski, I. \& Simberloff, D., 2008: A ticking time-bomb? The small Indian mongoose in Europe. Aliens 26, 14-16.

Barun, A., Hanson, C.C., Campbell, K.J. \& Simberloff, D., 2011a: A review of small Indian mongoose management and eradication on islands. In: Vertch, C.R; Clout, M.N. \& Towns, D.R. (eds.), Islands invasive: eradication and management. pp. 17-25.

Barun, A., Simberloff, D., Tvrtković, N. \& PAscal, M., 2011b: Impacts of the introduced small Indian mongoose (Herpestes auropunctatus) on abundance and activity time of the introduced ship rat (Rattus rattus) and the small mammal community on Adriatic islands, Croatia. NeoBiota 11, 51-61. 
Biedrzycka, A., Zalewski, A., Bartoszewicz, M,, Okarma, H. \& Jędrzejewska, E., 2014: The genetic structure of raccoon introduced in Central Europe reflects multiple invasion pathways. Biol. Invasions 16, 1611-1625.

Birnbaum, C., 2013: NOBANIS - Invasive Alien Species Fact Sheet Ondatra zibethicus. From: Online Database of the European Network on Invasive Alien Species NOBANIS (www.nobanis.org). Date of access 12/01/2018.

Bogdanovic, T., 2014: New data on racoon, Procyon lotor (Linnaeus, 1758) in Croatia. In: Jelaska, S. D. (ed.) Book of Abstracts of the $1^{\text {st }}$ Croatian symposium on invasive species with international participation. Croatian Ecological Society, Zagreb, pp. 19-20.

Bojović, D., 1992: Rođeni osvajač novih teritorija. Trag 8-9, 45.

Boršić, I., BorovečKi-VoskA, Lj., KutlešA, P. \& ŠEMničKI, P., 2015: New localities of Heracleum mantegazzianum Sommier et Levier (Apiaceae) in Croatia and control measures taken. Periodicum biologorum 117(3), 449-452.

Boršić, I., Milović, M., Dujmović, I., Bogdanović, S., Cigić, P., Rešetnik, I., Nikolić, T. \& Mitić, B., $2008:$ Preliminary check-list of invasive alien plant species (IAS) in Croatia. Natura Croatica 17(2), 55-71.

Bringsøe, H., 2006: NOBANIS - Invasive Alien Species Fact Sheet - Trachemys scripta. - From: Online Database of the North European and Baltic Network on Invasive Alien Species - NOBANIS (www. nobanis.org). Date of access 12/01/2018.

Brummitt, R. K., 1968: Heracleum L. In: Tutin, T. G., Heywood, V. H., Burges, N. A., Moore, D. M., Valentine, D. H., Walters, S. M. \& Webb, D. A. (eds.): Flora Europaea 2: Rosaceae to Umbelliferae. Cambridge University Press, Cambridge, pp. 364-366.

Budinski, I., Selanec, I. \& Kodžoman, A., 2018: Analiza postojećih podataka s razradom metodologije kartiranja za strane i invazivne strane vrste sisavaca vezanih za kopnene ekosustave. Izvještaj. Udruga BIOM za Hrvatsku agenciju za okoliš i prirodu. pp. 31.

Burić, I., Koren, T., Lauš, B., ŠTiH, A. \& Zadravec, M., 2018: Analiza postojećih podataka s razradom metodologije kartiranja za strane i invazivne strane vrste vodozemaca i gmazova. Association Hyla, Zagreb, Izvješće za Hrvatsku agenciju za okoliš i prirodu.

CABI, 2018a: Invasive Species Compendium. Wallingford, UK: CAB International (www.cabi.org/isc). Date of access 10/01/2018.

CABI, 2018b: Asclepias syriaca (common milkweed) [original text by Teeling, C.]. In: Invasive Species Compendium. Wallingford, UK: CAB International (www.cabi.org/isc). Date of access 12/01/2018.

CABI, 2018c: Elodea nuttallii (Nuttall's waterweed) [original text by Duenas, M. A.]. In: Invasive Species Compendium. Wallingford, UK: CAB International (www.cabi.org/isc). Date of access 10/01/2018.

CABI, 2018d: Heracleum mantegazzianum (giant hogweed). In: Invasive Species Compendium. Wallingford, UK: CAB International (www.cabi.org/isc). Date of access 10/01/2018.

CABI, 2018e: Impatiens glandulifera (Himalayan balsam). In: Invasive Species Compendium. Wallingford, UK: CAB International (www.cabi.org/isc). Date of access 10/01/2018.

CABI, 2018f: Myriophyllum heterophyllum (broadleaf watermilfoil) [original text by THum, R. \& Zuelig, M.]. In: Invasive Species Compendium. Wallingford, UK: CAB International. (www.cabi.org/isc). Date of access 10/01/2018.

CABI, 2018g: Procambarus fallax f. virginalis (Marmorkrebs) (original text by Chucholl, C.). In: Invasive Species Compendium. Wallingford, UK: CAB International (www.cabi.org/isc on 31-01-2018). Date of access 15/01/2018.

CABI, 2018h: Perccottus glenii (Amur sleeper) [original text by Grabowska J.]. In: Invasive Species Compendium. Wallingford, UK: CAB International (www.cabi.org/isc). Date of access 27/07/2018.

CABI, 2018i: Pseudorasbora parva (topmouth gudgeon) [original text by Copp, G. \& Siriwardena, S.]. In: Invasive Species Compendium. Wallingford, UK: CAB International (www.cabi.org/isc). Date of access 27/07/2018.

CABI, 2018j: Trachemys scripta elegans (red-eared slider) [original text by IUCN SSC Invasive Species Specialist Group]. In: Invasive Species Compendium. Wallingford, UK: CAB International (www. cabi.org/isc). Date of access 27/07/2018.

CABI, 2018k: Nyctereutes procyonoides (raccoon dog) [original text by Kauhala, K.]. In: Invasive Species Compendium. Wallingford, UK: CAB International (www.cabi.org/isc). Date of access 15/01/2018.

CABI, 20181: Ondatra zibethicus (muskrat) [original text by: Triplet, P.]. In: Invasive Species Compendium. Wallingford, UK: CAB International (www.cabi.org/isc). Date of access 10/01/2018. 
CABI, 2018m: Procyon lotor (raccoon) [original text by: Gehrt, S.]. In: Invasive Species Compendium. Wallingford, UK: CAB International (www.cabi.org/isc). Date of access 10/01/2018.

CAEN (Croatian Agency for Environment and Nature), 2018: Croatian Agency for Environment and Nature database.

Callaghan, C. T. \& Brooks, D.M., 2016: Ecology, Behavior, and Reproduction of Invasive Egyptian Geese (Alopochen aegyptiaca) in Texas. Bulletin of the Texas Ornithological Society 49(1-2), 37-45.

Canova, L. \& Rossi, S., 2008: First records of the Northern raccoon Procyon lotor in Italy. Hystrix It. J. Mamm. 19(2), 179-182.

Capurro, M., Galli, L., Mori, M., Salvidio, S. \& Arillo, A., 2015: Reproductive cycle of Pacifastacus leniusculus (Dana) (Crustacea: Decapoda) from the Brugneto Lake (Liguria, northwest Italy). Italian Journal of Zoology 82(3), 366-377.

Carter, J., Foote, A.L. \& Johnson-Randall, L.A., 1999: Modeling the effects of nutria (Myocastor coypus) on wetland loss. Wetlands 19, 209-219.

CARTER, J. \& Leonard, B.P., 2002: A review of the literature on the worldwide distribution, spread of, and efforts to eradicate the coypu (Myocastor coypus). Wildlife Society Bulletin 30, 162-175.

Chucholl, C., Morawetz, K. \& Gross, H., 2012: The clones are coming - strong increase in Marmorkrebs (Procambarus fallax (Hagen, 1870) f. virginalis) records from Europe. Aquatic Invasions 7, 511-519.

СнuсноцL, C., 2014: Predicting the risk of introduction and establishment of an exotic aquarium animal in Europe: insights from one decade of Marmorkrebs (Crustacea, Astacida, Cambaridae) releases. Management of Biological Invasions 5(4), 309-318.

Cigić, P., Nikolić, T., Plazibat, M., Hršak, V. \& Jelaska, S. D., 2003: The distribution of the genus Impatiens L. (Balsaminaceae) in Medvednica Nature Park, Croatia. Natura Croatica 12(1), 19-29.

Соoк, C. D. K., 1968: Myriophyllum L. In: Tutin, T. G., Heywood, V. H., Burges, N. A., Moore, D. M., Valentine, D. H., Walters, S. M. \& Webb, D. A. (eds.), Flora Europaea 2: Rosaceae to Umbelliferae. Cambridge University Press, Cambridge, pp. 311-312.

Copp, G.H., Bianco, P.G., Bogutskaya, N., Erôs, T., Falka, I., Ferreira, M.T., Fox, M.G., Freyhof, J., Gozlan, R.E., Grabowska, J., Kováč, V., Moreno-Amich, R., Naseka, A.M., PeŇaz, M., Povž, M., Przybylski, M., Robillard, M., Russell, I.C., Stakénas, S., Šumer, S., Vilagispert, A. \& Wiesner, C. 2005: To be, or not to be, a non-native freshwater fish? Journal of Applied Ichthyology 21: 242-262.

Corbet, G. B. \& Hill J. E., 1992: The mammals of the Indomalayan region: A systematic review. Oxford University Press, Oxford.

Crandall, K.A., 2010: Procambarus fallax. The IUCN Red List of Threatened Species 2010: e. T153961A4569411. (http://dx.doi.org/10.2305/IUCN.UK.2010-3.RLTS.T153961A4569411.en). Date of access 19/01/2018.

Ćaleta, M., Jelić, D., Buj, I., Zanella, D., Marčić, Z., Mustafić, P. \& Mrakovčić, M., 2011: First record of the alien invasive species rotan (Perccottus glenii Dybowski, 1877) in Croatia. Journal of Applied Biology 27(1), 146-147.

Ćirović, D. \& Milenković, M., 1999: Previous findings of the raccoon dog (Nyctereutes procyonoides ussuriensis matschie, 1907) in Yugoslavia and analysis of probable paths of its immigration. Contributions to the Zoogeography and Ecology of the Eastern Mediterranean Region 1, 75-82.

Ćirović, D., Raković, M., Milenković, M. \& Paunović, M., 2011: Small Indian Mongoose Herpestes auropunctatus (Herpestidae, Carnivora): an invasive species in Montenegro. Biological Invasions 13, 393399.

Ćirović, D. \& ToholJ, D., 2015: Distribution of Small Indian Mongoose (Herpestes auropunctatus) in the Eastern Herzegovina - spreading inside Balkan mainlanad. Balkan Journal of Wildlife Reaserch 2(1), 33-37.

DAISIE, 2018: DAISIE European Invasive Alien Species Gateway (http://www.europe-aliens.org/). Date of access 15/01/2018.

DANELL, K., 1996: Introductions of aquatic rodents: lessons of the muskrat Ondatra zibethicus invasion. Wildlife biology 2, 213-220.

Dandy, J. E., 1980: Elodea. In: Tutin, T. G., Heywood, V. H., Burges, N. A., Moore, D. M., Valentine, D. H., Walters, S. M. \& Webb, D. A. (eds.), Flora Europaea 5: Alismataceae to Orchidaceae (Monocotyledones). Cambridge University Press, Cambridge, pp. 4-5.

Dolenec, F., 1934: Odantra u Jugoslaviji. Priroda 24, 207-211.

Duplić, A., SlijepćEvić, V., Popović, N. \& JedrišKo, P., 2016: First record of the raccoon dog (Nyctereutes procyonoides) in the Croatian parts of Dinarides. In: JeLAsKA, S. D. (ed.), Book of Abstracts of the 2nd 
Croatian symposium on invasive species with international participation. Croatian Ecological Society, Zagreb, p. 50.

ĐorĐević, S. \& AnĐelković, M., 2016: Possible reproduction of the red-eared slider, Trachemys scripta elegans (Reptilia: Testudines: Emydidae), in Serbia, under natural conditions. Hyla Herpetological Bulletin 1, 44-49.

EASIN (European Commission - Joint Research Centre), 2018: European Alien Species Information Network (EASIN) (https://easin.jrc.ec.europa.eu/). Date of access 15/01/2018.

EC, 1997: Council Regulation (EC) No 338/97 of 9 December 1996 on the protection of species of wild fauna and flora by regulating trade therein (consolidated version). Official Journal of the European Union L 061 3.3.1997, p. 1. https://eur-lex.europa.eu/legal-content/EN/TXT/?uri=CELEX:0199 7R0338-20170204

EC, 2016: Commission Implementing Regulation (EU) 2016/1141 of 13 July 2016 adopting a list of invasive alien species of Union concern pursuant to Regulation (EU) No 1143/2014 of the European Parliament and of the Council. Official Journal of the European Union L 189, 14.7.2016, pp. 4-8. http://eur-lex. europa.eu/legal-content/EN/TXT/?qid=1468477158043\&uri=CELEX:32016R1141

EC, 2017: Commission Implementing Regulation (EU) 2017/1263 of 12 July 2017 updating the list of invasive alien species of Union concern established by Implementing Regulation (EU) 2016/1141 pursuant to Regulation (EU) No 1143/2014 of the European Parliament and of the Council. Official Journal of the European Union L 182, 13.7.2017, pp. 37-39. http://eur-lex.europa.eu/legal-content/EN/ TXT/?uri=CELEX:32017R1263

EEA, 2016: Biogeographical regions. Europe 2016. (https://www.eea.europa.eu/data-and-maps/data/ biogeographical-regions-europe-3). Date of access 10/12/2018.

Elis, J.C., Shulman, M.J., Jessor, H., Suomala, R., Morris, S.R., Seng, V., Wagner, M., Mach, K., 2007: Impact of raccoons on breeding success in large colonies of Great Black Gulls and Herring Gulls. Waterbirds 30, 375-383.

EPPO, 2009: EPPO data sheet on Invasive Alien Plants: Heracleum mantegazzianum, Heracleum sosnowskyi and Heracleum persicum. EPPO Bulletin 39, 489-499.

EPPO, 2015: Pest risk analysis for Myriophyllum heterophyllum. EPPO, Paris. Available at http://www. eppo.int/QUARANTINE/Pest_Risk_Analysis/PRA_intro.htm.

EPPO, 2016: Myriophyllum heterophyllum Michaux. Bulletin OEPP/EPPO Bulletin 46(1), 20-24.

EU, 2014: Regulation (EU) 1143/2014 of the European Parliament and of the Council of 22 October 2014 on the prevention and management of the introduction and spread of invasive alien species. Official Journal of the European Union L 317, 4.11.2014, pp.35-55. http://eur-lex.europa.eu/legal-content/EN/ TXT/?qid=1417443504720\&uri=CELEX:32014R1143

Fabre, P.H., Galewski, T., Tilak, M.K. \& Douzery, E.J.P., 2013: Diversification of South American spiny rats (Echimyidae): A multigene phylogenetic approach. Zoologica Scripta 42, 117-134.

Franjić, J., TrinajSTIĆ, I. \& ŠKvorc, Ž., 1998: Prilog poznavanju širenja nekih neofita u Hrvatskoj. Fragmenta Phytomedica et Herbologica 26(1-2), 5-17.

Fratini, F., Turchi, B., Ebani, V. V, Bertelloni, F., Galiero, A. \& Cerri, D., 2015: The presence of Leptospira in coypus (Myocastor coypus) and rats (Rattus norvegicus) living in a protected wetland in Tuscany (Italy). Veterinarski Arhiv 85, 407-414.

García, J.T, García, FJ, Aldda F, González J.L, Aramburu M.J, Cortes Y, et al., 2012: Recent invasion and status of the raccoon (Procyon lotor) in Spain. Biol. Invasions 14, 1305-1310.

Gaubert, P., 2016: Fate of the Mongooses and the Genet (Carnivora) in Mediterranean Europe: None Native, All Invasive? In: Angelici, F.M. (ed.), A problematic wildlife. A Cross-Disciplinary Approach. Springer International Publishing Switzerland. pp. 295-314.

GeBHARDT, H., 1996: Ecological and economic consequences of introductions of exotic wildlife (birds and mammals) in Germany. Wildlife Biology 2, 205-211.

GeHRT, S.D., 2004: Ecology and management of striped skunks, raccoons, and coyotes in urban landscapes. In: Fascione, N., Delach, A., Smith, M. (eds.), Predators and people: from conflict to conservation. Island Press Washington, pp. 81-104.

Gilchrist, J.S., Jennings, A.P., Veron, G. \& Cavallini, P., 2009: Family herpestidae (mongooses). In: Wilson, D.E. \& MitTermeier, R.A. (eds.), Handbook of the Mammals of the World. Volume 1. Carnivores. Lynx, Barcelona. pp. 262-328.

GISD (Global Invasive Species Database), 2018: Species profile: Herpestes auropunctatus. (http://www. iucngisd.org/gisd/speciesname/Herpestes+javanicus). Date of access 31/01/2018. 
GJurašić, M. \& Benić Penava, M., 2014: Uvođenje stranih invazivnih vrsta i njihov utjecaj na zavičajni ekosustav: Primjer otoka Mljeta. Ekonomska i ekohistorija 10, 149-172.

Gosling, L.M., BaKer, S.J. \& Clarke, C.N., 1988: An attempt to remove coypus (Myocastor coypus) from a wetland habitat in East Anglia. Journal of Applied Ecology 25, 49-62.

Govedič, M., 2017: First record of the spiny-cheek crayfish (Orconectes limosus) in Slovenia - $300 \mathrm{~km}$ upstream from its known distribution in the Drava River. Knowledge and Management of Aquatic Ecosystems 418, 7.

Gozlan, R. E., Andreou, D., Asaeda, T., Beyer, K., Bouhadad, R., Burnard, D., Caiola, N., Cakic, P., Djikanovic, V., Esmaeili, H. R., Falka, I., Golicher, D., Harka, A., Jeney, G., Kovac, V., Musil, J., Nocita, A., Povz, M., Poulet, N., Virbickas, T., Wolter, C., Tarkan, A. S., Tricarico, E., Trichkova, T., Verreycken, H., Witkowski, A., Zhang, C. G., Zweimueller, I. \& Britton, J. R., 2010: Pan-continental invasion of Pseudorasbora parva: towards a better understanding of freshwater fish invasions. Fish and Fisheries 11(4), 315-340.

GRIIS, 2018: GRIIS Global Register of Introduced and Invasive Species (www.griis.org ). Date of access 10/01/2018.

GuAn, R.Z., 1994: Burrowing behaviour of signal crayfish, Pacifastacus leniusculus (Dana), in the River Great Ouse, England. Freshwater Forum 4(3), 155-168.

Guichón, M.L., Benítez, V.B., Aвba, A., Borgnia, M. \& Cassini, M.H., 2003: Foraging behaviour of coypus Myocastor coypus: Why do coypus consume aquatic plants? Acta Oecologica 24, 241-246.

Gyimesi, A. \& Lensink, R., 2010: Risk analysis of the Egyptian Goose in the Netherlands. Bureau Waardenburg bv / Ministry of Agriculture, Nature and Food Quality, Invasive Alien Species Team.

Habeković, D. \& Popović, J., 1991: Stanje i iskorištenost ribljeg fonda u rijeci Savi od Podsuseda do Strelečkog. Ribarstvo Jugoslavije 46, 1-9.

Halse, S., 1984: Diet, Body Condition, and Gut Size of Egyptian Geese. The Journal of Wildlife Management 48(2), 569-573.

Hartman, L.H., Gaston, A.J. \& Eastman, D.S., 1997: Raccoon predation on ancient murrelets on East Limestone Island, British Columbia. Journal of Wildlife Management 61, 377-388.

Hartman, L.H, \& EAstman, D.S., 1999: Distribution of introduced raccoons Procyon lotor on the Queen Charlotte Islands: implications for burrow-nesting seabirds. Biological Conservation 88, 1-13.

Hays W.S.T. \& Conant S., 2007: Biology and impacts of Pacific islands invasive species. 1. A worldwide review of effects of the small Indian mongoose, Herpestes javanicus (Carnivora: Herpestidae). Pacific Science 61(1), 3-16.

Heinzel H., Fitter, R. S. R. \& Parslow, J., 1995: Birds of Britain \& Europe with North Africa \& the Middle East. HarperCollins Publishers, London.

Hejda, M., 2009: Impatiens glandulifera Royle, Himalayan balsam (Balsaminaceae, Magnoliophyta) In: DAISIE (eds.), The Handbook of Alien Species in Europe Springer, Berlin. pp. 351.

HelmisaARI, H., 2010: NOBANIS - Invasive Alien Species Fact Sheet - Impatiens glandulifera. - From: Online Database of the European Network on Invasive Alien Species - NOBANIS (www.nobanis. org). Date of access 11/01/2018.

Herwood, V. H. (ed.), 1972: Asclepiadaceae. In: Tutin, T. G., Heywood, V. H., Burges, N. A., Moore, D. M., Valentine, D. H., Walters, S. M. \& Webb, D. A. (eds.), Flora Europaea 3: Diapensiaceae to Myoporaceae. Cambridge University Press, Cambridge. pp. 70-73.

Hirtz, M., 1937a: Vijesti: Nutrija u Međimurju i Sloveniji. Priroda 2, 59-61.

Hirtz, M., 1937b: Vijesti: Vlak pregazio neobičnu životinju. Priroda 9, 286-287.

Holdich, D. \& Black, J., 2007: The spiny-cheek crayfish, Orconectes limosus (Rafinesque, 1817) (Crustacea: Decapoda: Cambaridae), digs into the UK. Aquatic Invasions 2(1), 1-15.

HommA, S. \& Geiter, O., 2010: Movements of neozoan ("exotic") geese in Germany and the Netherlands tracked by ringing. SOVON Ganzen en zwanendag, Arnhem.

Hudina, S., Faller, M., Lucić, A., Klobučar \& G., Maguire, I., 2009: Distribution and dispersal of two invasive crayfish species in the Drava River basin, Croatia. Knowledge and Management of Aquatic Ecosystems 394-395, 9.

Hudina, S., Galić, N., Roessink, I. \& Hock, K., 2011: Competitive interactions between co-occurring invaders: identifying asymmetries between two invasive crayfish species. Biological Invasions 13, 17911803.

Hudina, S, Žganec, K, Lucić, A, Trgovčić, K \& Maguire, I., 2013: Recent invasion of the karstic river systems in Croatia through illegal introductions of the signal crayfish. Freshw Crayfish 19, 21-27. 
Hudina, S., Hock, K., Radović, A., Klobučar, G., Petrović, J., Jelić, M. \& Maguire, I., 2016: Speciesspecific differences in dynamics of agonistic interactions may contribute to the competitive advantage of the invasive signal crayfish (Pacifastacus leniusculus) over the native narrow-clawed crayfish (Astacus leptodactylus). Marine and Freshwater Behaviour and Physiology 49(3), 147-157.

Ikeda, T., Asano, M., Матова, Y., Аве, G., 2004: Present status of invasive alien raccoon and its impact in Japan. Global Environmental Research 8, 125-131.

ITIS, 2018: Herpestes. Retrieved from the Integrated Taxonomic Information System on-line database (http://www.itis.gov). Date of access 10/08/2018.

Janev Hutinec, B., Kletečki, E., Lazar, B., Podnar Lešić, M., Skejić, J., Tadić, Z. \& Tvrtković, N., 2006: Crvena knjiga vodozemaca i gmazova Hrvatske. State Institute for Nature Protection, Zagreb.

Jasprica, N., Lasić, A. L., Hafner, D. \& Bratoš Cetinić, A., 2017: European invasion in progress: Myriophyllum heterophyllum Michx. (Haloragaceae) in Croatia. Natura Croatica 26(1), 99-103.

JaszczoŁt, J. \& SzAniawska, A., 2011: The spiny-cheek crayfish Orconectes limosus (Rafinesque, 1817) as an inhabitant of the Baltic Sea - experimental evidences for its invasion of brackish waters. Oceanological and Hydrobiological Studies 40(3), 52-60.

Jelić, L., Janev Hutinec, B. \& Jelić, D., 2016: Reproductive biology of Trachemys scripta (Schoepff, 1792) in continental Croatia, In: Jelaska, S. D. (ed.), Book of Abstracts of the 2nd Croatian symposium on invasive species with international participation. Croatian Ecological Society, Zagreb, pp. 36.

Jelić, L. \& Jelić, D., 2015: Allochthonous species of Turtles in Croatia and Bosnia and Herzegovina. Hyla Herpetological Bulletin 1, 53-64.

Johnsen, S.I. \& TAugbøL, T., 2010: NOBANIS - Invasive Alien Species Fact Sheet - Pacifastacus leniusculus. - From: Online Database of the European Network on Invasive Alien Species - NOBANIS (www. nobanis.org). Date of access 15/01/2018.

Johnson, M.F., Rice, S.P. \& Ried, I., 2010: Topographic disturbance of subaqueous gravel substrates by signal crayfish (Pacifastacus leniusculus). Geomorphology 123, 269-278.

JosEFsSON, M., 2011: NOBANIS - Invasive Species Fact Sheet - Elodea canadensis, Elodea nuttallii and Elodea callitrichoides - From: Online Database of the European Network on Invasive Alien Species - NOBANIS (www.nobanis.org). Date of access 10/01/2018.

Kaman, M., 1950: Ondatra. Lovački vjesnik 59, 179-180.

Kauhala, K., 1996: Habitat use of raccoon dogs, Nyctereutes procyonoides, in southern Finland. Zeitschrift fur Saugetierkunde 61, 269-275.

Kauhala, K. \& KowalczyK, R., 2011: Invasion of the raccoon dog Nyctereutes procyonoides in Europe: History of colonization, features behind its success, and threats to native fauna. Current Zoology 57, 584-598.

Kauhala, K. \& KowalczyK, R., 2012: The raccoon dog (Nyctereutes procyonoides) in the community of medium-sized carnivores in Europe: its adaptations, impact on native fauna and management of the population. In: Carnivores: Species, conservation, and management. Nova Publisher New York, pp. 113-134.

Keller N.S., Pfeiffer M., Roessink I., Schulz R. \& Schrimp A., 2014: First evidence of crayfish plague agent in populations of the marbled crayfish (Procambarus fallax forma virginalis). Knowledge and Management of Aquatic Ecosystems 414, 15.

Kirbiš, N., Bedjanič, M., Kus Veenvliet, J., Veenvliet, P., Stanković, D., Lipovšek, G. \& Poboljšaj, K., 2016: First records of the American bullfrog Lithobates catesbeianus (Shaw, 1802) in Slovenia. Natura Sloveniae 18(1), 23-27.

Klingenstein, F., 2007: NOBANIS - Invasive Alien Species Fact Sheet - Heracleum mantegazzianum. From: Online Database of the North European and Baltic Network on Invasive Alien Species NOBANIS (www.nobanis.org). Date of access 10/01/2018.

Kočić, A., Horvatić, J. \& Jelaska, S. D., 2014: Distribution and morphological variations of invasive macrophytes Elodea nuttallii (Planch.) H. St. John and Elodea canadensis Michx in Croatia. Acta Botanica Croatica 73(2), 437-446.

Koren, T., Štih, A., Burić, I., Koller, K., Lauš, B. \& Zadravec, M., 2018: The current distribution of pond slider Trachemys scripta (Reptilia: Emydidae) in Croatia. Natura Sloveniae 20(1), 33-44.

Koščo, J., Manko, P., Miklisova, D. \& Košuthova, L., 2008: Feeding ecology of invasive Perccottus glenii (Perciformes, Odontobutidae) in Slovakia. Czech Journals of Animal Science 53(11): 479-486.

Kottelat, M. \& Freyhof, J., 2007: Handbook of the European Freshwater Fishes. Publications Kottelat, Cornol, Switzerland. 
Kouba, A., Petrusek, A. \& KozÁK, P., 2014: Continental-wide distribution of crayfish species in Europe: update and maps. Knowledge and Management of Aquatic Ecosystems 413, 05.

KowalczyK, R., 2006: NOBANIS - Invasive Alien Species Fact Sheet Nyctereutes procyonoides. From: Online Database of the European Network on Invasive Alien Species - NOBANIS (www.nobanis.org). Date of access 15/01/2018.

KozÁk, P., Buřič, M., Policar, T., HamačKova, J. \& Lepičova, A., 2007: The effect of inter- and intraspecific competition on survival and growth rate of native juvenile noble crayfish Astacus astacus and alien spiny-cheek crayfish Orconectes limosus. Hydrobiologia 590(1), 85-94.

Kralj, J. \& BARIšIć, S., 2013: Rare birds in Croatia. Third report of the Croatian Rarities Committee. Natura Croatica 22(2), 375-396.

Krausch, H.-D., 1996: Farbatlas Wasser- und Uferpflanzen. Ulmer, Stuttgart.

Kryštufek, B. \& Tvrtković, N., 1992: New information on the introduction into Europe of the small Indian mongoose, Herpestes auropunctatus. Small Carnivore Conservation 7, 16.

Kume, M., Onoda, Y., Negishi, J.N., Sagawa, S., Nagayama, S. \& Kayaba, Y., 2012: Feeding damage by exotic species, nutria (Myocastor coypus), to unionid mussels in a floodplain water-body of the Kiso River, Japan. Biology of Inland Waters 27, 41-47.

Lever, C., 2005: Naturalised Birds of the World. T \& A.D. Poyser, London.

Lewis, S.D., 2002: Pacifastacus. In: Holdich D. M. (Ed.). Blackwell Science, Oxford: 511-540.

Linscombe, G. \& Kinler, N., 1997: A survey of vegetative damage caused by nutria herbivory in the Barataria and Terrebonne Basins. Barataria-Terrebonne National Estuary Program 31, 1-17.

Lókkös, A., Müller, T., Kovács, K., Várkonyi, L., Specziár, A., Martin, P., 2016: The alien, parthenogenetic marbled crayfish (Decapoda: Cambaridae) is entering Kis-Balaton (Hungary), one of Europe's most important wetland biotopes. Knowledge and Management of Aquatic Ecosystems 417, 16.

Loss, A.C., MourA, R.T. \& LeITE, Y.L.R., 2014: Unexpected phylogenetic relationships of the painted tree rat Callistomys pictus (Rodentia: Echimyidae). Natureza 12, 132-136.

Lotze, J.-H. \& Anderson, S., 1979: Procyon lotor. Mammalian Species 19, 1-8.

Lowe, S., Browne, M., Boudjelas, S. \& De Poorter, M., 2000: 100 of the World's Worst Invasive Alien Species A selection from the Global Invasive Species Database. The Invasive Species Specialist Group (ISSG) a specialist group of the Species Survival Commission (SSC) of the World Conservation Union (IUCN), First published as special lift-out in Aliens 12, December 2000. Updated and reprinted version: November 2004. IUCN, Auckland, New Zealand.

Luca, M. \& GhiorghițĂ, G., 2014: The invasive species Perccottus glenii - a threat to the fresh water ecosystems. Analele Ştiințifice ale Universității „Alexandru Ioan Cuza” din Iași, s. Biologie animală, Tom LX.

Luca, N. De, Kovačıć, D. \& Đulić, B., 1989: Fauna vodozemaca, gmazova i sisavaca NP “Krka." In: Kerovec, M. (ed.), Zbornik radova s Simpozija "NP Krka” - stanje istraženosti i problemi zaštite ekosistema. Ekološke Monografije 2, 523-550.

Lukács, B. A., Mesterházy, A., VidéKI, R. \& KirÁly, G., 2016: Alien aquatic vascular plants in Hungary (Pannonian ecoregion): Historical aspects, data set and trends. Plant Biosystems - An International Journal Dealing with all Aspects of Plant Biology 150(3), 388-395.

LukAČ, G., 1989: Nova nalazišta vrste Impatiens glandulifera Royle u Hrvatskoj. Acta Botanica Croatica 48, 165-168.

Lukač, M., Pedersen, K. \& Prukner-Radovčić, E., 2015: Prevalence of Salmonella in captive reptiles from Croatia. Journal of Zoo and Wildlife Medicine 46(2), 234-240.

Lyко, F., 2017: The marbled crayfish (Decapoda: Cambaridae) represents an independent new species. Zootaxa 4363(4), 544-552.

Maguire, I., Jelić, M. \& KlobučAR, G., 2011: Update on the distribution of freshwater crayfish in Croatia. Knowledge and Management of Aquatic Ecosystems 401, 31.

Maguire, I. \& Klobučar, G., 2003: Appearance of Orconectes limosus in Croatia. Crayfish News 3(25), 7.

Maguire, I., Klobučar, G., Marčić, Z. \& Zanella, D., 2008: The first record of Pacifastacus leniusculus in Croatia. Crayfish News 30(4), 4.

Maguire, I., Klobučar, G., Žganec, K., Jelić, M., Lucić, A. \& Hudina, S., 2018: Recent changes in distribution pattern of freshwater crayfish in Croatia - threats and perspectives. Knowl. Managt. Aquatic Ecosyst. 419, 2. 
Mangnall, M. J. \& Crowe, T. M., 2002: Population dynamics and the physical and financial impacts to cereal crops of the Egyptian Goose Alopochen aegyptiacus on the Agulhas Plain, Western Cape, South Africa. Agriculture, Ecosystems \& Environment 90(3), 231-246.

Marković, Lj., 1970: Prilozi neofitskoj flori savskih obala u Hrvatskoj. Acta Botanica Croatica 29, $203-211$.

Marković, LJ., 1984: Zur Verbreitung und Vergesellschaftung von Impatiens glandulifera in Kroatien. Acta botanica Slovaca Academiae Scientiarum Slovacae. Series A, Taxonomica, geobotanica. Suppl. 1, 209215.

Marzano, F.N., Scalici, M, Chiesa, S., Gherardi, F., Piccinini, A., Gibertini, G., 2009: The first record of the marbled crayfish adds further threats to fresh waters in Italy. Aquatic Invasions 4, 401-404.

Martin, P., Dorn, N., Kawai, T., van der Heiden, C. \& Scholtz, G., 2010a: The enigmatic Marmorkrebs (marbled crayfish) is the parthenogenetic form of Procambarus fallax (Hagen, 1870). Contributions to Zoology 79, 107-118.

Martin, P., Shen, H., Füller, G. \& Scholtz, G., 2010b: The first record of the parthenogenetic Marmorkrebs (Decapoda, Astacida, Cambaridae) in the wild in Saxony (Germany) raises the question of its actual threat to European freshwater ecosystems. Aquatic Invasions 5, 397-403

Martin, P., Thonagel, S. \& Scholtz, G., 2016: The parthenogenetic Marmorkrebs (Malacostraca: Decapoda: Cambaridae) is a triploid organism. Journal of Zoological Systematics and Evolutionary Research 54, 13-21.

Maslo, S., 2010: Giant hogweed Heracleum mantegazzianum Somier \& Levier - a new non-indigenous species in the flora of Bosnia and Herzegovina. Herbologia 11(2), 17-24.

MAsLo, S., 2016: Preliminary list of invasive alien plant species (IAS) in Bosnia and Herzegovina. Herbologia 16(1), 1-14.

Michel, V., Ruvoen-Clouet, N., Menard, A., Sonrier, C., Fillonneau, C., Rakotovao, F., Ganière, J.P. \& André-Fontaine, G., 2001: Role of the coypu (Myocastor coypus) in the epidemiology of leptospirosis in domestic animals and humans in France. European Journal of Epidemiology 17, 111-121.

Mitić, B., Boršić, I., Dujmović, I., Bogdanović, S., Milović, M., Cigić, P., RešEtnik, I., Nikolić, T., 2008 : Alien flora of Croatia: proposals for standards in terminology, criteria and related database. Natura Croatica 17(2), 73-90.

Moutou, F., 1997: Aquatic and semi-aquatic mammals introduced into France. Risks and Consequences. Bulletin Francais De La Peche Et De La Piscicultur 344/345, 133-139.

Nagano, N., OAna, S., Nagano, Y. \& Arakawa, Y., 2006: A severe Salmonella enterica serotype paratyphi B infection in a child related to a pet turtle, Trachemys scripta elegans. Japanese Journal of Infectious Diseases 59(2), 132-134.

Nehring, S. \& Steinhof, J., 2015: First records of the invasive Amur sleeper, Perccottus glenii Dybowski, 1877 in German freshwaters: a need for realization of effective management measures to stop the invasion. BioInvasions Records 4(3), 223-232.

Nellis, D.W., 1989: Herpestes auropunctatus. Mammalian species 342, 1-6.

Nellis, D.W. \& Everard, C.O.R., 1983: The biology of the mongoose in the Caribbean. Studies on the Fauna of Curaçao and other Caribbean Islands 1, 1-162.

Nikolić, T., Mitić, B. \& Boršıć, I., 2014: Flora Hrvatske - invazivne biljke. Alfa d. d., Zagreb

Nikolić, T. (ed.), 2018: Flora Croatica Database. On-Line (http://hirc.botanic.hr/fcd). Department of Botany and Botanical Garden, Faculty of Science, University of Zagreb, Zagreb. Date of access 13/06/2018.

NOBANIS, 2015: Invasive Alien Species: Pathway Analysis and Horizon Scanning for Countries in Northern Europe. Nordic Council of Ministers, Copenhagen.

NOBANIS, 2018: European Network on Invasive Alien Species, Gateway to Information on Invasive Alien species in North and Central Europe (http://www.NOBANIS.org). Date of access 12/01/2018.

Novitsky, R.A. \& Son, M.O., 2016: The first records of Marmorkrebs (Procambarus fallax (Hagen, 1870) f. virginalis) (Crustacea, Decapoda, Cambaridae) in Ukraine. Ecologica Montenegrina 5, 44-46.

NowAK, E., 1984: Development of populations and geographical distribution of the raccoon dog Nyctereutes procyonoides (Gray, 1834) in Europe. Z. Jagdwissenschaft 30, 137-154.

Oivanen, L., Kapel, C.M.O., Pozio, E., Rosa, G. La, Mikkonen, T. \& Sukura, A., 2002: Associations between Trichinella species and host species in Finland. The Journal of Parasitology 88, 84-88.

Oksanen, A., Lindgren, E. \& Tunkkari, P., 1998: Epidemiology of trichinellosis in lynx in Finland. Journal of Helminthology 72, 47-53. 
Owen, C.T., Mcgregor, M.A., Cobbs, G.A. \& Alexander, J.E., 2011: Muskrat predation on a diverse unionid mussel community: Impacts of prey species composition, size and shape. Freshwater Biology 56, 554-564.

Pandža, M., Franjić, J., Trinajstić, I., ŠKvorc, Ž. \& STANČić, Z., 2001: The most recent state of affairs in the distribution of some neophytes in Croatia. Natura Croatica 10(4), 259-275.

Panzacchi, M., Cocchi, R., Genovesi, P., Bertolino, S., Cocehi, R. \& Genovesi, P., 2007: Population control of coypu Myocastor coypus in Italy compared to eradication in UK: a cost-benefit analysis. Wildlife Biology 13, 159-171.

Patou, M.L., Mclenachan, P.A., Morley, C.G., Couloux, A., Jennings, A.P. \& Veron, G., 2009: Molecular phylogeny of the Herpestidae (Mammalia, Carnivora) with a special emphasis on the Asian Herpestes. Molecular Phylogenetics and Evolution 53, 69-80.

Pavletić, Z., 1993: Prilog rasprostranjenosti vrste Impatiens glandulifera Royle (Balsaminaceae) u Hrvatskoj. Fragmenta Phytomedica et Herbologica 21(1), 63-66.

Perez-Santigosa, N., Díaz-Paniagua, C. \& Hidalgo-Vila, J., 2008: The reproductive ecology of exotic Trachemys scripta elegans in an invaded area of southern Europe. Aquatic Conservation: Marine and Freshwater Ecosystems 18(7), 1302-1310.

Petrova, A., Vladimirov, V. \& Georgiev, V., 2013: Invasive alien species of vascular plants in Bulgaria. Institute of Biodiversity and Ecosystem Research, Bulgarian Academy of Sciences, Sofia

Pimentel, D., 1955: Biology of the Indian mongoose in Puerto Rico. Journal of Mammalogy 36, 62-68.

Piria, M., Povž, M., Vilizzi, L., Zanella, D., Simonović, P. \& Copp, G. H., 2016: Risk screening of nonnative freshwater fishes in Croatia and Slovenia using the Fish Invasiveness Screening Kit. Fisheries Management and Ecology 23, 21-31.

Pitman, C. R. S., 1965: The nesting and some other habits of Alopochen, Nettapus, Plectropterus and Sarkidiornis. Wildfowl Trust Ann. Rep. 16, 115-121.

Plančıć, J., 1937: Ondatra (bizamski štakor) - pojava, način života, rasprostranjenje i način tamanjenja. Zavod za primijenjenu zoologiju Savske Banovine, Zagreb.

Preston, C. D. \& Croft, J. M., 1997: Aquatic plants in Britain and Ireland. Harley Books, Colchester, UK.

Puкy, M. \& ScháD, P., 2006: Oronectes limosus colonises new areas fast along the Danube in Hungary. Bull. Fr. Pêche Piscic. 380-381, 919-926.

Purger, J.J. \& KryštufeK, B., 1991: Feral coypu Myocastor coypus (Rodentia, Mammalia) in Yugoslavia. Biološki Vestnik 39, 19-24.

Pyšek, P., Danihelka, J., Sádlo, J., Chrtek, J. Jr., Chytrý, M., Jarošík, V., Kaplan, Z., Krahulec, F., Moravcová, L., Pergl, J., Štajerová, K. \& Tichý, L., 2012: Catalogue of alien plants of the Czech Republic (2nd edition): checklist update, taxonomic diversity and invasion patterns. Preslia 84, 155-255.

Reshetnikov, A. N. \& Schliewen, U. K., 2013: First record of the invasive alien fish rotan Perccottus glenii Dybowski, 1877 (Odontobutidae) in the Upper Danube drainage (Bavaria, Germany). Journal of Applied Ichthyology 29(6), 1367-1369.

Reshetnikov, A. N. \& Ficetola, G. F., 2011: Potential range of the invasive fish rotan (Perccottus glenii) in the Holarctic. Biological Invasions 13, 2967-2980.

Reshetnikov, A. N., 2013: Spatio-temporal dynamics of the expansion of rotan Perccottus glenii from West-Ukrainian centre of distribution and consequences for Eurpean freshwater ecosystems. Aquatic Invasions 8(2), 193-206.

Richardson, D. M., Pyšek, P., Rejmánek, M., Barbour, M. G., Panetta, F. D. \& West, C. J., 2000: Naturalization and invasion of alien plants: concepts and definitions. Diversity and Distributions 6, 93-107.

Samardžić, M., Lucić, A., Maguire, I. \& Hudina, S., 2014: The First Record of the Marbled Crayfish (Procambarus fallax (Hagen, 1870) f. virginalis) in Croatia. Crayfish News 36(4), 4.

SAvić, I.R., 1962: Fluktuacija brojnosti ondatre (Ondatra zibethica L.) u Jugoslaviji. 11. Kongres biologa Jugoslavije, Beograd, pp. 166-167.

Schweiger, M., 2015: First record of breeding of the alien turtle species Trachemys scripta elegans in the wild on the island of Krk, Croatia? Hyla Herpetological Bulletin 1, 50-52.

Scotт, D.A. \& Rose, P.M., 1996: Atlas of Anatidae Populations in Africa and Western Eurasia. Wetlands International Publication No. 41. Wetlands International,Wageningen, The Netherlands, pp. 91-93.

Selanec, I., Budinski, I. \& Kodžoman, A., 2016: Procjena brojnog stanja i utvrđivanje metodologije za kontrolu širenja i iskorjenjivanje invazivne strane vrste Herpestes javanicus auropunctatus - mungos, na otoku Mljetu. Pages 53-70. In: Duplić, A. \& Budinski, I., 2016: Procjena veličine početnog stanja i utvrđivanje metodologije za kontrolu širenja i iskorjenjivanje sljedećih invazivnih stranih vrsta (eng. 
invasive alien species, IAS): 1. Ailanthus altissima (Mill.) Swingle - pajasen, 2. Herpestes javanicus auropunctatus - mungos, 3. Leporidae sp. - kunić/zec na otocima. Final report. Karlovac University of Applied Sciences and Association BIOM. pp 70.

Shinmanter, E., Weisman, Y., Lublin, A., Mechani, S., Gruenberg, R., Horowith, H. \& Lipkind, M., 1998: Avian Paramyxoviruses Serotype 3 Isolated from Captive Birds in Israel: Clinical Signs, Pathology, and Antigenic Characterization. Avian Diseases 42(2), 418-422.

Simberloff, D., Dayan, T., Jones C. \& Ogura, G., 2000: Character displacement and release in the small Indian mongoose, Herpestes javanicus. Ecology 81(8), 2086-2099.

Singer, A., Kauhala, K., Holmala, K. \& Smith, G.C., 2009: Rabies in northeastern Europe-the threat from invasive raccoon dogs. Journal of Wildlife Diseases 45, 1121-1137.

SKyrienĖ, G. \& Paulauskas, A., 2012: Distribution of invasive muskrats (Ondatra zibethicus) and impact on ecosystem. Ekologija 58, 357-367.

Smirnov, V. V. \& Tretyakov, K., 1998: Changes in aquatic plant communities on the island of Valaam due to invasion by the muskrat Ondatra zibethicus L. (Rodentia, Mammalia). Biodiversity and Conservation 7, 673-690.

Smith, K. G. \& Fames, D. A., 2012: History and Current Status of Egyptian Goose (Alopochen aegyptiacus) in Northwestern Arkansas. Journal of the Arkansas Academy of Science 66, Article 36, 200-204.

SöDERBÄCK, B., 1991: Interspecific dominance relationship and aggressive interactions in the freshwater crayfishes Astacus astacus (L.) and Pacifastacus leniusculus (Dana). Canadian Journal of Zoology 69(5), 1321-1325.

SöDERвÄск, B., 1992: Predator avoidance and vulnerability of two co-occurring crayfish species, Astacus astacus (L.) and Pacifastacus leniusculus (Dana). Annales Zoologici Fennici 29(4), 253-259.

Sorvillo, F., Ash, L., Berlin, O. et al., 2002: Basylisascaris procyonis: And emerging helminthic zoonosis. Emerging Infectious Diseases 8, 355-359.

StarmüHLeR, W., 2009: Vorarbeiten zu einer „Flora von Istrien“, Teil XII. Carinthia II, 199./119., 553-600.

Steppan, S.J. \& SchenK, J.J., 2017: Muroid rodent phylogenetics: 900-Species tree reveals increasing diversification rates. PLoS ONE 12, e0183070.

Stewart. F. \& Grace, J., 1984: An experimental study of hybridization between Heracleum mantegazzianum Somm. \& Levier and H. sphondylium L. subsp. sphondylium (Umbelliferae). Watsonia 15, 73-83.

Stunković, H., 2009: Heracleum mantegazzianum Somier \& Levier - field observations. In: Nikolıć, T. (ed.) Flora Croatica Database. Faculty of Science, Zagreb (http://hirc.botanic.hr/fcd). Date of access 10/01/2018.

Sutor, A., Kauhala, K. \& Ansorge, H., 2010: Diet of the raccoon dog Nyctereutes procyonoides - a canid with an opportunistic foraging strategy. Acta Theriologica 55, 165-176.

ŠKraba, D., Tosić, A., Miličić, D., Nikolić, V. \& Simonović, P., 2013: Invasiveness assessment of the Chinese mitten crab Eriocheir sinensis (H. Milne Edwards, 1853) in the Serbian section of the River Danube. Archives of Biological Sciences 65, 353-358.

Šver, L., McConnell, J., Krušić Tomaić, I., Petrović, M., Tomaić, J. \& Gužvica, G., 2018: Raccoon dog in Croatia: individual occurrrences or established population? In: Jelaska, S. D. (ed.), Book of Abstracts of the $3^{\text {rd }}$ Croatian symposium on invasive species with international participation. Croatian Ecological Society, Zagreb, p. 66.

TAYLOR, M.E. \& MATHESON, J., 1999: A craniometrics comparison of the African and Asian mongooses in the genus Herpestes (Carnivora: Herpestidae) Mammalia 63, 449-464.

Trichkova, T., Todorov, M., Hubenov, Z. \& Jurajda, P., 2015: First record of Orconectes limosus (Rafinesque, 1817) in Bulgaria. Esenias Tools (project websites http://www.esenias.org/index. php?option=com_content\&task=view\&id=366).

Trinajstić, I., 1974: Ein neuer Fundort von Impatiens glandulifera Royle in Kroatien. Fragmenta Herbologica Jugoslavica 40, 1-6.

TrinajSTiĆ, I. \& Franjić, J., 1994: Prilog poznavanju rasprostranjenosti vrste Impatiens glandulifera Royle (Balsaminaceae) u Hrvatskoj. Fragmenta Phytomedica et Herbologica 22(1-2), 21-24.

Trinajstić, I. \& Franjić, J., 1995: As. Impatienti-Solidaginetum M. Moor 1958 (Calistegion sepium) u vegetaciji Republike Hrvatske. Fragmenta Phytomedica et Herbologica 23(1), 25-30.

Tvrtкović, N., 1985: Ribe Jugoslavije. HAZU, Zagreb.

TvrtKović, N. \& KryštufeK, B., 1990: Small Indian mongoose Herpestes auropunctatus (Hodgson, 1836) on the Adriatic Islands of Yugoslavia. Bonner Zoologische Beitrage 41, 3-8. 
Usio, N., Konishi, M. \& NaKano, S., 2001: Species Displacement Between an Introduced and A 'vulnerable' Crayfish: The Role of Aggressive Interactions and Shelter Competition. Biological Invasions 3(2), 179-185.

Veitch, C. R., \& Clout, M. N. (eds.), 2002: Turning the tide: The Eradication of Invasive Species: Proceedings of the International Conference on Eradication of Island Invasives. Occasional Paper of the IUCN Species Survival Commission No. 27, IUCN.

Veron, G., Patou, M.L., Рothet, G., Simberloff, D. \& Jennings A.P., 2007: Systematic status and biogeography of the Javan and small Indian mongoose (Herpestidae, Carnivora). Zoologica Scripta 36, 1-10.

Veron, G. \& Jennings A., 2017: Javan mongoose or small Indian mongoose - who is where? Mammalian Biology 87, 62-70.

VIdović, M., 2013: Elodea nuttallii (Planch.) H.St.John - field observations. In: Nikolić, T. (ed.): Flora Croatica Database. Faculty of Science, Zagreb (http://hirc.botanic.hr/fcd). Date of access 10/01/2018.

Vidović, M., 2015: Elodea nuttallii (Planch.) H.St.John - field observations. In: Nikolić, T. (ed.): Flora Croatica Database. Faculty of Science, Zagreb (http://hirc.botanic.hr/fcd). Date of access 10/01/2018.

Vogt, G., 1999: Diseases of European freshwater crayfish, with particular emphasis on interspecific transmission of pathogens. In: GHerardi, F. \& Holdich, D. M. (eds.), Crayfish in Europe as alien species. How to make the best of a bad situation? A.A. Balkema, Rotterdam, Brookfield, pp. 87-103.

Vogt, G., Tolley, L., \& Scholtz, G., 2004: Life Stages and Reproductive Components of the Marmorkrebs (Marbled Crayfish), the First Parthenogenetic Decapod Crustacean. Journal of morphology 261, 286311.

Vojkovská, R., Horka, I. \& Ďuriš, Z., 2014: The diet of the spiny-cheek crayfish Orconectes limosus in the Czech Republic. Central European Journal of Biology 9(1), 58-69.

Walters, S. M., 1968: Impatiens L. In: Tutin, T. G., Heywood, V. H., Burges, N. A., Moore, D. M., Valentine, D. H., Walters, S. M. \& Webb, D. A. (eds.), Flora Europaea 2: Rosaceae to Umbelliferae. Cambridge University Press, Cambridge, pp. 240-241.

WARD, O.G. \& Wurster-Hill, D., 1990: Nyctereutes procyonoides. Mammalian Species 358, 1-5.

Welcomme, R. L., 1988: International introductions of inland aquatic species. FAO Fisheries Technical Paper 294.

Wenzel, E. \& Haltenorth, T., 1972: System der Schleichkatzen (Viverridae). Säugetierkunde Mitteilungen 20, 110-127.

Willner, G.R., Feldhammer, G.A., Zucker, E.E. \& Chapman, J.A., 1980: Ondatra zibethicus. Mammalian Species 141, 1-8.

Wilson, D. E. \& Reeder, D. M. (eds.) 2005: Mammal Species of the World. A Taxonomic and Geographic Reference (3rd ed), Johns Hopkins University Press.

Windhoffer, E., 2017. Nutria Aggr [Video File] (https://www.youtube.com/watch?v=_E3BpoDuNrg\&feature=youtu.be). Date of access 10/12/2018.

Wiткоwski, A., 2011: NOBANIS - Invasive Alien Species Fact Sheet - Pseudorasbora parva. - From: Online Database of the European Network on Invasive Alien Species - NOBANIS (www.nobanis.org). Date of access 15/01/2018.

Woods, C.A., Contreras, L., Willner-Chapman, G. \& Whidden, H.P., 1992: Myocastor coypus. Mammalian Species 398, 1-8.

Wozencraft, W.C., 2005: Order Carnivora. In: Wilson, D.E. \& ReEder, D.M. (eds.), Mammal Species of the World: A taxonomic and geographic reference. Third Edition, Smithsonian Institution Press, Washington, DC, USA. pp. 532-628.

Yalçin-Özdilek, Ş., Kirankaya, Ş. G. \& EкMEKçI, F. G., 2013: Feeding ecology of the topmouth gudgeon Pseudorasbora parva (Temminck and Schlegel, 1846) in the Gelingüllü Reservoir, Turkey. Turkish Journal of Fisheries and Aquatic Sciences 13, 87-94.

Zalewski, A., 2011: GB Non-native Organism Risk Assessment for Procyon lotor. (www.nonnativespecies. org).

Zaninović, K., Gajić-ČAPKA, M., PerčEc TAdić, M. et al., 2008: Climate atlas of Croatia 1961-1990., 19712000. Državni hidrometeorološki zavod, Zagreb. 


\title{
SAŽETAK
}

\section{Invazivne strane vrste koje izazivaju zabrinutost u Uniji (Uredba 1143/2014) prisutne u Hrvatskoj}

\author{
I. Boršić, A. Ješovnik, T. Mihinjač, P. Kutleša, S. Slivar, M. Cigrovski Mustafić \\ \& S. Desnica
}

Uredba 1143/2014 o sprječavanju i upravljanju unošenja i širenja invazivnih stranih vrsta određuje okvir za sprječavanje, svođenje na najmanju moguću mjeru i ublažavanje štetnih učinaka invazivnih stranih vrsta na bioraznolikost u Europskoj uniji. Iako je Uredba obvezujuća i mora se u cijelosti primjenjivati u svim državama članicama, njezina implementacija u Hrvatskoj osigurava se Zakonom o sprječavanju unošenja i širenja stranih te invazivnih stranih vrsta i upravljanju njima („Narodne novine“, br. 15/18).

Uredba se odnosi na invazivne strane vrste u Europskoj uniji te se sukladno Uredbi donosi popis invazivnih stranih vrsta koje izazivaju zabrinutost u Uniji ("Unijin popis"). Prvi popis donesen je Provedbenom uredbom Komisije 2016/1141 i uključivao je 37 vrsta. Dodatnih 12 vrsta dodano je na Unijin popis Provedbenom uredbom Komisije 2017/1263. Od tih 49 vrsta, 17 vrsta prisutno je u Hrvatskoj. One uključuju pet biljaka (Plantae): Asclepias syriaca L., Elodea nuttallii (Planch.) St. John, Heracleum mantegazzianum Sommier \& Levier, Impatiens glandulifera Royle i Myriophyllum heterophyllum Michaux.; tri raka (Crustacea): Orconectes limosus (Rafinesque, 1817), Pacifastacus leniusculus (Dana, 1852) i Procambarus fallax (Hagen, 1870) f. virginalis; dvije slatkovodne ribe (Actinopterygii): Perccottus glenii (Temminck \& Schlegel, 1846) i Pseudorasbora parva Dybowski, 1877: jednog gmaza (Reptilia): Trachemys scripta (Schoepff, 1792); jednu pticu (Aves): Alopochen aegyptiacus (Linnaeus, 1766); i pet sisavaca (Mammalia): Herpestes javanicus (É. Geoffroy Saint-Hilaire, 1818), Myocastor coypus (Molina, 1782), Nyctereutes procyonoides (Gray, 1834), Ondatra zibethicus (Linnaeus, 1766) i Procyon lotor (Linnaeus, 1758).

Dodatno, tri biljne vrste s Unijinog popisa ne nalaze se na popisu flore Hrvatske, niti su prema našem saznanjima prisutne u prirodi, ali je moguće da su u Hrvatskoj prisutne kao ukrasne biljke u uzgoju. To su: Eichhornia crassipes (Martius) Solms, Ludwigia peploides (Kunth) P.H. Raven i Pennisetum setaceum (Forssk.) Chiov. Dvije dodatne životinjske vrste s Unijinog popisa koje u radu spominjemo su Eriocheir sinensis (Malacostraca) i Lithobates catesbeianus (Amphibia). One su prisutne u susjednim zemljama te su zabilježene i u blizini hrvatske granice, pa je njihov unos u budućnosti vjerojatan.

U radu predstavljamo sistematiku i biologiju vrsta s Unijinog popisa prisutnih u Hrvatskoj, prirodno područje rasprostranjenosti, područje unosa, povijest unosa, putove njihovog unosa u Hrvatsku, utjecaj u unesenom području, uključujući i poznate utjecaje u Hrvatskoj. Također, temeljem dostupnih podataka prikazujemo njihovu rasprostranjenost u Hrvatskoj.

Neke od vrsta s Unijinog popisa prisutne u Hrvatskoj poznate su samo s jednog ili nekoliko nalazišta, dok su druge široko rasprostranjene. Ovisno o njihovoj prisutnosti u Hrvatskoj odnosno njihovoj rasprostranjenosti, Uredba propisuje prevenciju, rano otkrivanje i brzo iskorjenjivanje, ili upravljanje. Dakle, za uspješnu borbu protiv invazivnih stranih vrsta koje izazivaju zabrinutost u Uniji prisutnih u Hrvatskoj, a kako bi se spriječio ili ublažio njihov štetan učinak, bit će potrebni znatni napori, uz suradnju i usklađeno djelovanje s ostalim sektorima (npr. šumarstvo, poljoprivreda, ribarstvo, upravljanje vodama, granična kontrola). 